\title{
Combining Cep290 and Mkks ciliopathy alleles in mice rescues sensory defects and restores ciliogenesis
}

\author{
Rivka A. Rachel, ${ }^{1}$ Helen L. May-Simera, ${ }^{2}$ Shobi Veleri, ${ }^{1}$ Norimoto Gotoh, ${ }^{1}$ Byung Yoon Choi, ${ }^{3}$ \\ Carlos Murga-Zamalloa, ${ }^{4}$ Jeremy C. Mclntyre, ${ }^{5}$ Jonah Marek, ${ }^{6}$ Irma Lopez, ${ }^{6}$ Alice N. Hackett, ${ }^{1}$ \\ Matthew Brooks, ${ }^{1}$ Anneke I. den Hollander,7 Philip L. Beales, ${ }^{8}$ Tiansen Li, ${ }^{1}$ Samuel G. Jacobson, ${ }^{9}$ \\ Raman Sood, ${ }^{10}$ Jeffrey R. Martens, ${ }^{5}$ Paul Liu, ${ }^{10}$ Thomas B. Friedman, ${ }^{3}$ Hemant Khanna, ${ }^{4}$ \\ Robert K. Koenekoop, ${ }^{6}$ Matthew W. Kelley, ${ }^{2}$ and Anand Swaroop' \\ ${ }^{1}$ Neurobiology Neurodegeneration and Repair Laboratory, National Eye Institute, NIH, Bethesda, Maryland, USA. \\ ${ }^{2}$ Laboratory of Cochlear Development and ${ }^{2}$ Laboratory of Molecular Genetics, National Institute on Deafness and Other Communication Disorders, NIH, \\ Bethesda, Maryland, USA. ${ }^{4}$ Department of Ophthalmology and Visual Sciences and 5Department of Pharmacology, University of Michigan, \\ Ann Arbor, Michigan, USA. ${ }^{6}$ McGill Ocular Genetics Laboratory and Pediatric Ophthalmology, McGill University Health Centre, Montreal, Quebec, Canada. \\ ${ }^{7}$ Departments of Ophthalmology and Human Genetics, Radboud University Nijmegen Medical Centre, Nijmegen, The Netherlands. \\ ${ }^{8}$ Molecular Medicine Unit, UCL Institute of Child Health, London, United Kingdom. 9Department of Ophthalmology, Scheie Eye Institute, \\ University of Pennsylvania, Philadelphia, Pennsylvania, USA. ${ }^{10}$ Genetics and Molecular Biology Branch, \\ National Human Genome Research Institute, NIH, Bethesda, Maryland, USA.
}

Cilia are highly specialized microtubule-based organelles that have pivotal roles in numerous biological processes, including transducing sensory signals. Defects in cilia biogenesis and transport cause pleiotropic human ciliopathies. Mutations in over 30 different genes can lead to cilia defects, and complex interactions exist among ciliopathy-associated proteins. Mutations of the centrosomal protein $290 \mathrm{kDa}$ (CEP290) lead to distinct clinical manifestations, including Leber congenital amaurosis (LCA), a hereditary cause of blindness due to photoreceptor degeneration. Mice homozygous for a mutant Cep290 allele (Cep290 ${ }^{\text {rd16 }}$ mice) exhibit LCA-like early-onset retinal degeneration that is caused by an in-frame deletion in the CEP290 protein. Here, we show that the domain deleted in the protein encoded by the Cep290 ${ }^{\text {rd } 16}$ allele directly interacts with another ciliopathy protein, MKKS. MKKS mutations identified in patients with the ciliopathy BardetBiedl syndrome disrupted this interaction. In zebrafish embryos, combined subminimal knockdown of $m k k s$ and $c e p 290$ produced sensory defects in the eye and inner ear. Intriguingly, combinations of Cep290rd16 and $M k k s^{k o}$ alleles in mice led to improved ciliogenesis and sensory functions compared with those of either mutant alone. We propose that altered association of CEP290 and MKKS affects the integrity of multiprotein complexes at the cilia transition zone and basal body. Amelioration of the sensory phenotypes caused by specific mutations in one protein by removal of an interacting domain/protein suggests a possible novel approach for treating human ciliopathies.

\section{Introduction}

Cilia and centrosomes are highly specialized microtubule-based organelles that are crucial mediators of diverse biological processes, including establishment of polarity and sensory signal transduction $(1,2)$. Primary cilia are ubiquitous, polarized, and nonmotile organelles that originate from the mother centriole (called the basal body) in postmitotic cells and serve as antennae for sensing biochemical signals $(3,4)$. Biogenesis of primary cilia and associated signaling events is coordinated by anterograde and retrograde intraflagellar transport (IFT) that uses kinesin and dynein motors, respectively (5). The primary cilium reportedly mediates the selective trafficking of as many as 1,000 proteins $(6,7)$.

Defects in primary cilia are associated with pleiotropic human disorders, collectively termed ciliopathies, which include a highly penetrant photoreceptor degeneration phenotype (8-11). Mutations in over 30 distinct genes that encode centrosomal cilia proteins lead

Authorship note: Rivka A. Rachel and Helen L. May-Simera contributed equally to this work.

Conflict of interest: The authors have declared that no conflict of interest exists. Citation for this article: J Clin Invest. 2012;122(4):1233-1245. doi:10.1172/JCI60981. to ciliopathies, including McKusick-Kaufmann syndrome (MKKS; MIM no. 236700), Joubert syndrome (JBTS; MIM no. 21330), Meckel-Gruber syndrome (MKS; MIM no. 249000), Bardet-Biedl syndrome (BBS; MIM no. 209900), nephronophthisis (NPHP), and Leber congenital amaurosis (LCA; MIM no. 611755). Multiple gene defects leading to overlapping clinical manifestations (12), modifier roles of specific alleles $(13,14)$, and discovery of macromolecular functional complexes (15-17) suggest dynamic interactions among proteins involved in ciliopathies. Interactions among MKS, NPHP, JBTS, and other ciliopathy proteins are required for basal body anchoring and establishment of a ciliary gateway (18). Similarly, IFT proteins interact to maintain ciliary architecture and transport (19). Disruptions of these protein networks, particularly at the base of the ciliary transition zone, result in defects of cilia biogenesis $(19,20)$ and signal transduction pathways (20-22). Hence, dissection of ciliary protein complexes and of unique interactions among their individual components should enhance our understanding of ciliopathies, allowing better design of therapeutic strategies.

Mutations in the centrosomal cilia protein CEP290 are responsible for up to $25 \%$ of LCA (23). A recent selective screen of 13 LCA genes in 60 affected subjects revealed a striking $43 \%$ of the mutant 
A

MKKS mutations

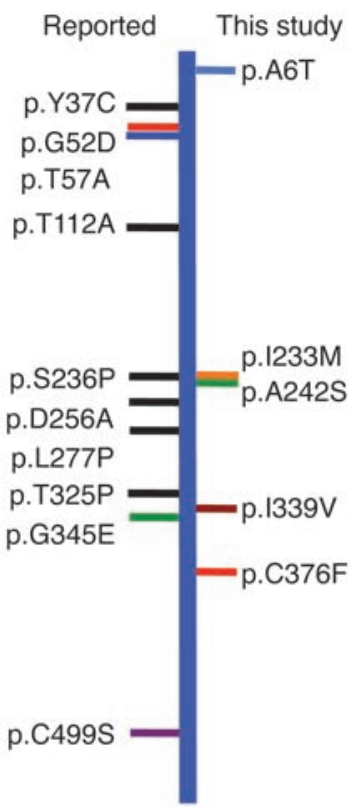

\section{B}
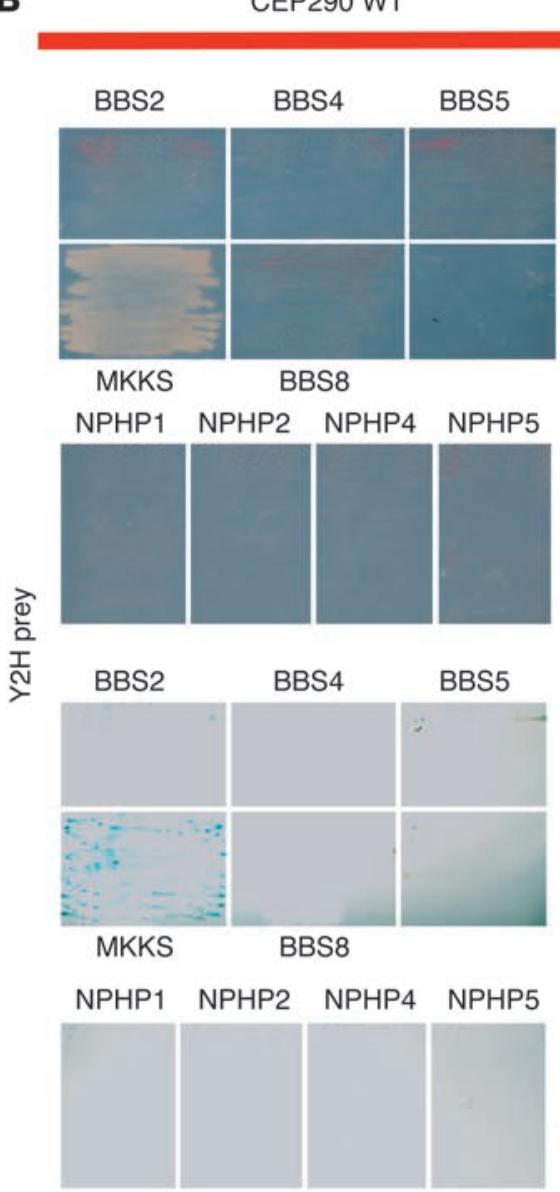

DSD (bait)

CEP290 WT

p53 + control
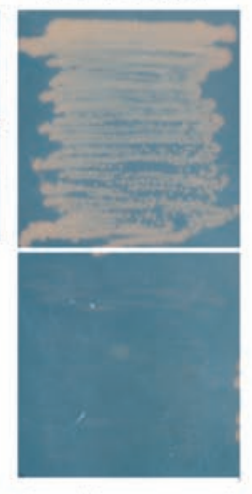

Laminin - control

p53 + control

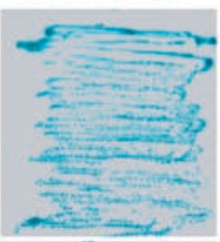

Laminin - control

\section{C}

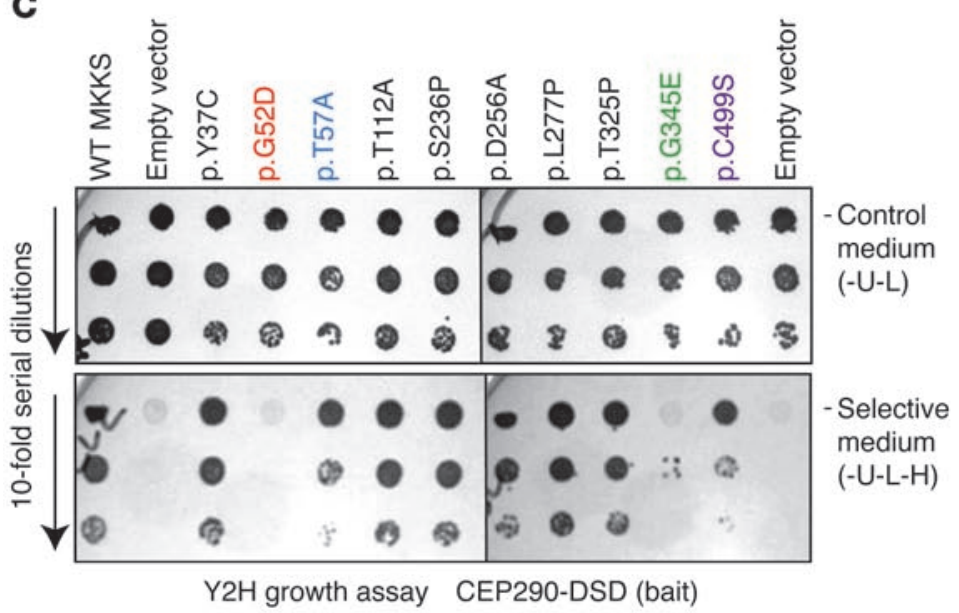

D

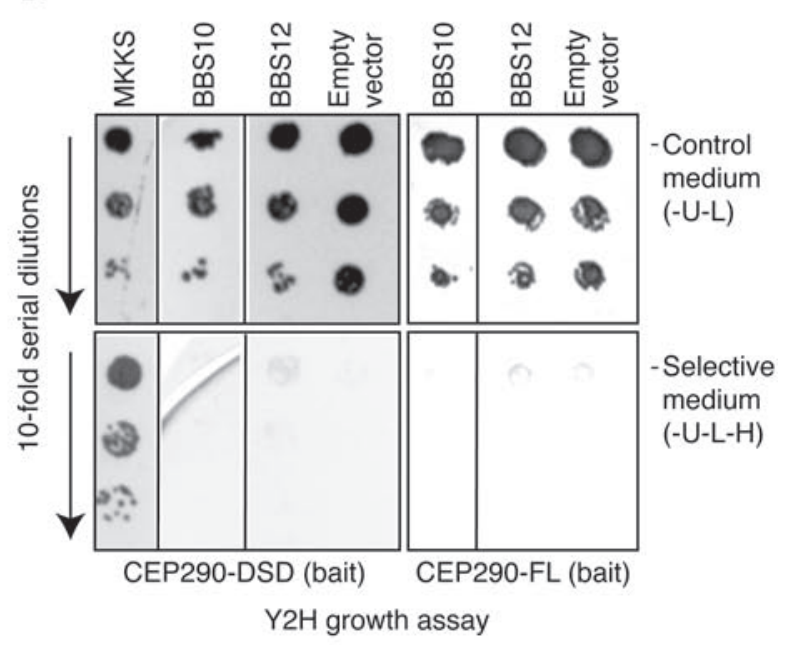




\section{Figure 1}

The CEP290-DSD domain specifically interacts with MKKS. (A) A schematic of the MKKS protein, with horizontal lines showing variants/mutations identified in this study (right) and previously reported (left). New sequence variants cluster in the same regions as known mutations. (B) Yeast 2-hybrid (Y2H) screen using the Cep290-DSD domain as bait identified MKKS as a specific interactor. p53/T-antigen and laminin/T-antigen combinations were positive and negative controls, respectively. Growth was detected with the MKKS prey only (top panels) and validated by $\beta$-galactosidase assay (bottom panels). (C) Interaction of the Cep290-DSD domain with mutant MKKS constructs in yeast 2-hybrid growth assay. The top 3 rows show growth of 10 -fold serial cell dilutions on double-selection control media (-U-L); the bottom 3 rows show growth on selective media (-U-L-H). Note equal growth on control media plates (top 3 rows). MKKS mutations at residues 52, 57, 345, and 499 disrupt interaction with CEP290. (D) Yeast 2-hybrid assay using CEP290-DSD (left 4 lanes) or full-length CEP290 (CEP290-FL) (right 3 lanes) as bait with full-length MKKS, BBS10, BBS12, or empty vector as prey. The top 3 rows show growth of 10 -fold serial cell dilutions on double-selection control media (-U-L); the bottom 3 rows show growth of 10 -fold serial dilutions on selective media (-U-L-H). Only MKKS interacts with Cep290-DSD. The first row of the BBS12 lane on selective medium shows a shadow due to dead cells.

alleles in Cep290 (24), suggesting a significant contribution of this gene to retinal disease manifestation. In addition, mutations in Cep290 produce varying clinical outcomes of JBTS (25-27), MKS (28-30), and BBS (31). Cep290 has been implicated in ciliogenesis $(32,33)$, which requires docking of the basal body to the plasma membrane and assembly of the BBSome (15) and chaperonin complexes (17). Moreover, in Chlamydomonas and C. elegans, cep290 is shown to localize to the transition zone of the cilia and help in establishing a gatekeeping function that regulates ciliary protein trafficking $(18,34,35)$.

During our mutation screening of patients with LCA, we recently observed variants in the ciliopathy gene MKKS (also known as BBS6) in almost $10 \%$ of affected individuals. As Cep290 provides the largest mutation load to LCA, we hypothesized a functional interaction of MKKS with CEP290 in the ciliary transition zone. Here, we report a direct physical association between these 2 proteins and demonstrate the physiological relevance of this interaction in mediating cilia biogenesis and function in 3 sensory systems. Our studies reveal a reciprocal functional rescue of visual, auditory, and olfactory phenotypes by specific mutant alleles of Cep290 and Mkks and suggest a novel paradigm for the treatment of ciliopathies.

\section{Results}

Identification of MKKS mutations in patients with LCA. As part of an effort to understand the phenotypic variability in inherited retinal degenerations, we have been examining patients with LCA (36) for mutations in $B B S$ and other ciliopathy-related genes. Sequencing the exons of a BBS gene, MKKS $(37,38)$, in 142 LCA probands led to identification of 13 heterozygous sequence variants (Figure 1A, Supplemental Figure 1, Supplemental Methods, and Supplemental Tables 1 and 2; supplemental material available online with this article; doi:10.1172/JCI60981DS1). Of these, 5 variants were predicted to be pathogenic in LCA based on in silico analyses (SIFT, Polyphen, and Blossum62), protein conservation, and their presence in patients with BBS. These variants were not detected in at least 100 normal ethnically matched controls and in the 1,000 Genome database (Supplemental Tables 3 and 4). Two of the vari- ants have previously been reported in patients with $\operatorname{BBS}(39,40)$. The identification of a novel, potentially pathogenic MKKS variant (p.C376F) in a patient carrying 2 CEP290 mutations (p.C998X and p.R1508X) further validates the suggested role of modifiers in ciliopathies $(13,14,24)$. Discovery of multiple Mkks missense variants in LCA, together with the suggested role of a chaperonin complex containing MKKS in ciliogenesis (17), prompted us to explore the possibility of biochemical and functional interactions between CEP290 and MKKS.

Direct interaction of Mkks with the Cep290-DSD domain that is deleted in a mouse model of LCA. In mice, a recessive Cep290 rd16 mutant allele that results in deletion of 299 residues (from amino acids 1599-1897; NP_666121) in the CEP290 protein is associated with severe LCA-like photoreceptor degeneration and olfactory dysfunction $(41,42)$. When used as bait in yeast 2-hybrid assays, the Cep290 ${ }^{\text {rd16 }}$ deleted region (termed deleted in sensory dystrophy [DSD] domain) interacted with full-length MKKS as prey (Figure 1B). Eight other cilia-associated proteins (BBS2, BBS4, BBS5, BBS8, and NPHP1, NPHP2, NPHP4, and NPHP5) did not reveal any obvious interaction with the DSD domain (Figure 1B). Evaluation of MKKS mutants/variants identified in human patients with BBS (Figure 1A) revealed that the p.G52D and p.G345E mutations eliminated the interaction with CEP290-DSD bait, while 2 others (p.T57A and p.C499S) reduced this association (Figure 1C), using yeast 2-hybrid assays. All mutant clones grew well on control medium (Figure 1C). Subsequent yeast 2-hybrid analyses did not uncover an interaction of either CEP290-DSD or full-length CEP290 with BBS10 or BBS12, the 2 known binding partners of MKKS (ref. 17 and Figure 1D). Coimmunoprecipitation experiments using transfected HEK293 cells (Figure 2, A and $\mathrm{B}$ ) or bovine retina extracts (Figure 2C) validated the interaction of CEP290 with MKKS. As in the yeast 2-hybrid assays with CEP290 and MKKS human mutant plasmids (Figure 1C), human $M K K S$ mutations altered or abrogated the biochemical interaction between in vitro transfected CEP290 and MKKS (Figure 2A). We note that differences in protein association between the yeast 2-hybrid and coimmunoprecipitation data may result from different expression levels of mutant plasmids as well as other factors intrinsic to yeast or HEK293 cells.

Cooperative effect of cep290 and mkks during zebrafish eye and ear development. To confirm and assess the physiological relevance of cep290 and mkks in vivo, we used translation-blocking antisense morpholinos $(27,43)$ to knockdown cep290 and mkks proteins in zebrafish embryos. We first established the minimal dosages of cep290 (1 ng) and mkks (2 ng) morpholinos that gave rise to ciliary phenotypes (refs. 27, 43-45, and Figure 3C). Coinjection into zebrafish embryos of subminimal dosages of the 2 morpholinos (0.5 ng cep290 and 1.0 ng mkks) that did not show ciliary anomalies on their own resulted in significant eye and inner ear defects (smaller eye size, altered retinal lamination, and defective inner ear tether cell development) as well as axis abnormalities $(17.8 \%, n=37$ out of 208 morphants, compared with $6.5 \%, n=16$ out of 245 uninjected; $P<0.001$ ) (Figure 3, A-C). Together, these results are consistent with defects in cilia function $(33,46,47)$. These studies in zebrafish suggest a functional synergy between CEP290 and MKKS during normal retinal and otocyst development.

Reduced retinal ciliopathy phenotypes in mice by combinations of Cep290 rd16 and Mkks ${ }^{k o}$ alleles. We then examined the interaction of Cep290 and Mkks in the corresponding mouse models of LCA and BBS, respectively. Mice with either a Cep290 rd16/rd16 or Mkks ${ }^{k o / k o}$ geno- 
A

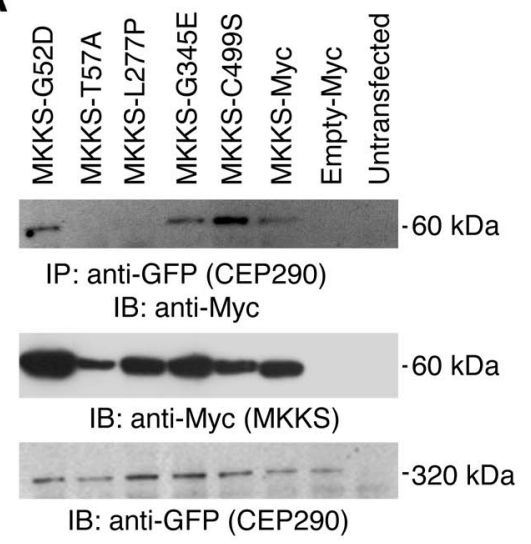

C

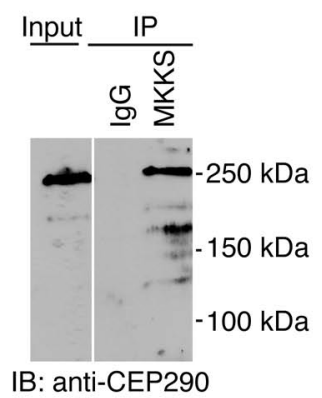

B
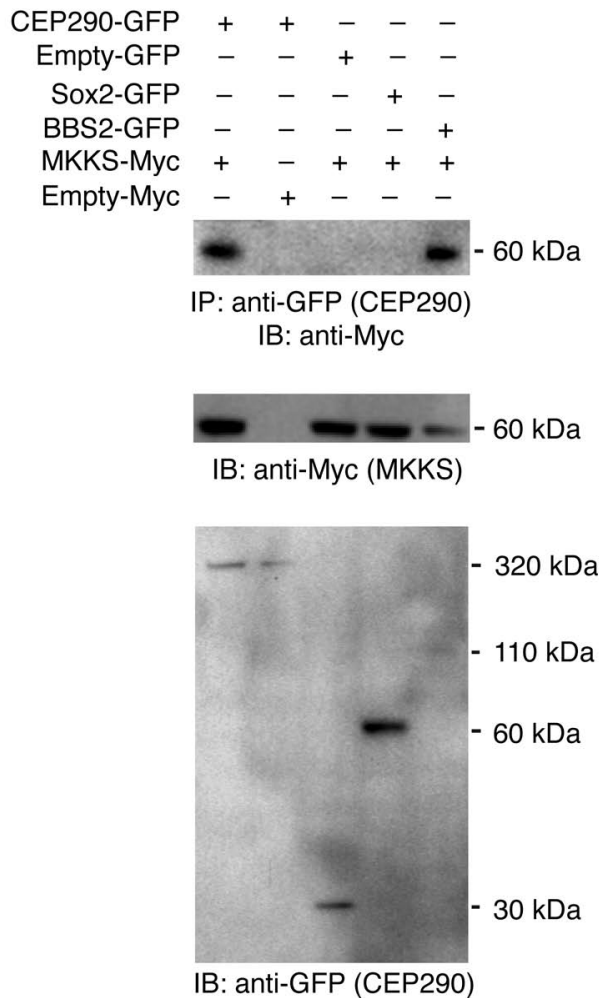

\section{Figure 2}

Coimmunoprecipitation of CEP290 and MKKS in transfected cells and from retinal extracts. (A) Coimmunoprecipitation of CEP290-GFP (full length) and MKKS-myc, expressed in transfected HEK293 cells. Some BBS-associated amino acid substitutions in MKKS disrupt coimmunoprecipitation with CEP290 (top). Expression of mutant MKKS protein is demonstrated by immunoblot analysis of the input for MKKS-Myc constructs (middle). CEP290 expression is shown by bands in input lanes (bottom). (B) Coimmunoprecipitation of WT MKKS-Myc with CEP290-GFP (full length) and negative control plasmids. Sox2 is used as a negative control and BBS2 is used as a positive control. Myc plasmids (middle) and GFP plasmids (bottom) were used as inputs. (C) Coimmunoprecipitation of CEP290 and MKKS from bovine retinal extracts. Immunoblots of proteins immunoprecipitated with anti-MKKS antibody or normal IgG were probed with anti-CEP290 antibody. Note that specific 290-kDa CEP290-immunoreactive protein is pulled down by the anti-MKKS antibody but not by control lgG. type have significant retinal deficits $(41,48,49)$. However, rather than displaying more severe phenotypes, mice carrying a combination of 3 mutant Cep290 rd16 and Mkks ${ }^{k o}$ alleles demonstrated lesser phenotypic defects in retinal photoreceptor cilia (Figure 4, A-C, and E). Mice with triallelic combinations (Cep290 rd16/rd16; $M k k s^{k o /+}$ or Cep290 ${ }^{r d 16 /+} ; M k k s^{k o / k o}$ mice) had reduced pathological lesions in the retinal fundi (Supplemental Figure 2) and better photoreceptor outer segment (OS) morphology (Figure 4A) and ultrastructure (Figure 4E) compared with the corresponding single or double homozygotes. Consistent with these findings, electroretinography (ERG) responses in triallelic mice at P20 also showed considerable improvement in comparison with those of either Cep290 rd16/rd16 or Mkksko/ko homozygotes (Figure 4C). The Cep290rd16/rd16;Mkks $s^{k o / k o}$ double-homozygous mutants exhibited a greater degree of phenotypic variability, even among littermates or between the 2 eyes, from nearly normal fundi to severe retinal pigmentary changes (Supplemental Figure 2), with no ERG improvement (Figure 4C). We then performed ultrastructural analysis of the photoreceptor connecting cilium, identifying structural features from the basal body through the ciliary axoneme (Figure 5, A and B). The flattened, oval appearance of the connecting cilium in the $M k k s^{k o / k o}$ genotype was significantly restored toward the normal round profile in the Cep290 ${ }^{r d 16 /+} ; M k k s^{k o / k o}$ genotype (Figure 5C). Cep290 rd16/rd16;Mkksko/ko double mutants revealed an abnormal basal body and connecting cilium in photoreceptors (Figure 5B and Supplemental Figure 3).

Rescue of ciliary defects in cochlear hair cells and olfactory sensory neurons in mice with combinations of Cep290rd16 and Mkks ko alleles. The Cep290 rd16/rd16 and Mkks ${ }^{k o / k o}$ mice also displayed both auditory (Figure 6D) and olfactory deficits $(41,48,49)$. In the cochlea, the development of actin-based stereocilia bundles on mechanosen- sory hair cells is regulated by the kinocilium, a single transient microtubule-based cilium (50). Morphological defects observed in stereociliary bundles of Cep290 rd16/rd16 or Mkks ${ }^{k o / k o}$ mutants were largely rescued in Cep290rd16/rd16;Mkks ko/ko double homozygotes (Figure 6A). Detailed analyses using higher-magnification images revealed increased length of hair cell kinocilia in Cep290rd16/rd16 mice and severe structural abnormalities in $M k k s^{k o / k o}$ mice at P0 (Figure 6, B and C). In contrast, cilia length and structure were completely rescued in Cep290 ${ }^{\text {rd16/rd16; } M k k s^{k o / k o}}$ double mutants (Figure $6, \mathrm{~B}$ and $\mathrm{C}$ ). Consistent with these results, auditory function, as assessed by auditory brainstem responses (ABRs), was abnormal in both Cep290 rd16/rd16 and Mkks ${ }^{k o / k o}$ homozygotes (Figure 6D) but was largely rescued in triple allelic Cep290rd16 and Mkks $s^{k o}$ combination mutants. Double homozygotes showed an intermediate phenotype that was less severe than that of either of the single homozygous mutants (Figure 6D and Supplemental Figure 4). Scanning EM of the olfactory epithelium at 8 weeks of age revealed morphologically normal cilia in all genotypes except Mkks ${ }^{k o / k o}$, in which few cilia were detectable at this age (see Figure 6E, white arrows; note that cilia appeared normal by immunostaining at P14 in Mkkso/ko mice; Supplemental Figure 5). Addition of 1 or 2 Cep290 2 rd16 alleles to $M k k^{k o / k o}$ genotype produced a normal pattern of olfactory cilia, even at 8 weeks of age (Figure 6E).

Adjacent subcellular localization of Cep290 and Mkks proteins in sensory cells. To further elucidate the mechanism of reciprocal rescue observed with Cep290 rd16 and Mkks so alleles, we examined the subcellular localization of CEP290 and MKKS in sensory neurons. In photoreceptors, CEP290 was localized just distal to the basal body in the connecting cilium (Figure 7A), consistent with previous reports $(27,41)$, whereas MKKS was primarily present in the basal 
A
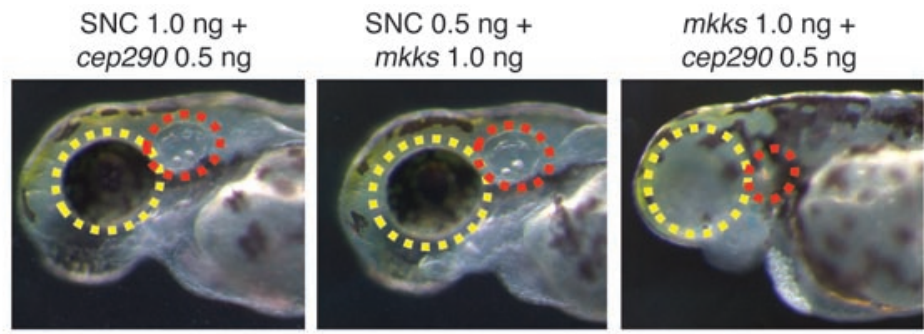

B

SNC $1.0 \mathrm{ng}+$ cep290 $0.5 \mathrm{ng}$

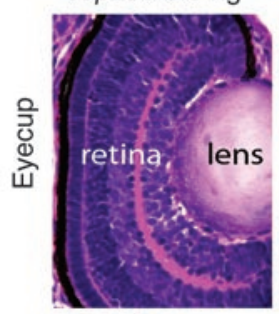

mkks $1.0 \mathrm{ng}+$

C
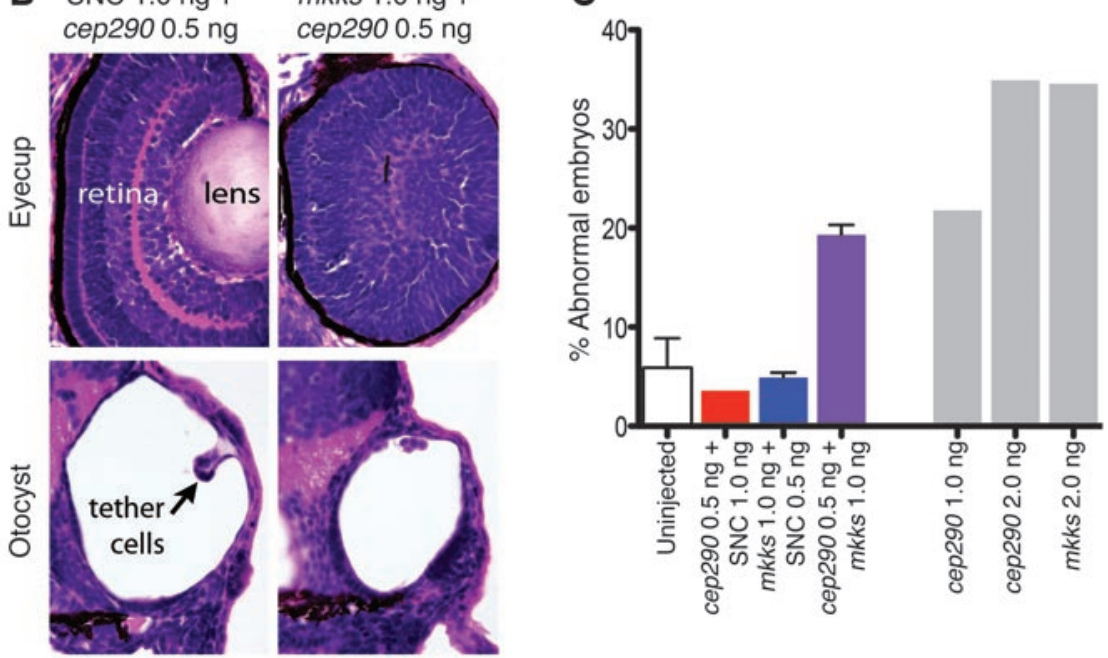

Figure 3

Knockdown by coinjection of subminimal doses of cep290 and mkks morpholinos causes sensory defects in zebrafish embryos. (A) Whole mount embryos at 72 hours after fertilization. Subminimal doses of morpholinos against cep290 and mkks transcripts and standard negative control (SNC) produced no phenotype, whereas coinjecting subminimal doses of $m k k s$ and cep290 resulted in deformed eyes (yellow circles) and ears (red ovals). Original magnification, $\times 11.5$. (B) H\&E-stained sections of eyecups and otocysts in control $(0.5 \mathrm{ng}$ cep290 plus $1.0 \mathrm{ng}$ SNC) and experimental ( $0.5 \mathrm{ng}$ cep290 plus $1.0 \mathrm{ng} \mathrm{mkks}$ ) embryos. Note the lack of normal lens formation and retinal lamination in the double morphant embryos (top panel) and impaired tether cell development in the otocyst (bottom panel). Original magnification, $\times 60$. (C) Quantitation of morphant phenotypes. Subminimal doses of cep290 or mkks morpholino alone resulted in abnormal embryos at a frequency similar to that of uninjected controls $(<5 \%)$, whereas combining both morpholinos yielded $18 \%$ of embryos with ear, eye, and/or axis defects. Higher doses of either morpholino alone revealed a greater percentage of defective embryos, consistent with previous results $(27,43,44)$. Eye or ear defects were never observed in uninjected embryos. Error bars are SD. Numbers of embryos examined per condition are 245 (uninjected), 53 (0.5 ng cep290 plus $1.0 \mathrm{ng}$ SNC), 121 (0.5 ng mkks plus $1.0 \mathrm{ng}$ SNC), 208 (0.5 ng cep290 plus $1.0 \mathrm{ng} \mathrm{mkks),} 98$ (1.0 ng cep290), 125 (2.0 ng cep290), and 55 (2.0 ng mkks).

body (Figure 7A). CEP290 and MKKS were adjacent but appear not to overlap (Figure 7B). We also detected the expression of CEP290 protein in Cep290rd16/rd16 and Mkksko/ko and in the double-mutant photoreceptors, even though the structures of the connecting cilia and inner segments were disrupted (Figure 7C).

In outer hair cells of $\mathrm{P} 0$ mouse cochlea, $\mathrm{CEP} 290$ localized distal to the basal body at the base of the kinocilium (Figure 7D), a true cilium that regulates development of the stereociliary bundle (50). In these cells, MKKS expression overlaps with $\gamma$-tubulin, a basal body marker (51), suggesting that MKKS is expressed in the basal bodies. In olfactory sensory neurons of P15 WT mice, CEP290 was detected just distal to $\gamma$-tubulin in the sensory cilia (Figure 7E). Thus, despite differences in the structure and function of the cilia in distinct sen- sory tissues, CEP290 is present in the proximal cilium, a region corresponding to the transition zone, in each cell type (52).

To further explore the functional consequences of the Cep290rd16 and Mkks ${ }^{k o}$ alleles, we localized other photoreceptor and/or cilia proteins, including PCM1 (53) and AHI1 (ref. 14 and Supplemental Figure 6). PCM1 accumulates at the outer limiting membrane in mutant genotypes, whereas expression of AHI1 is not significantly altered. Rhodopsin appears to accumulate in the inner segments and outer nuclear layer in the Cep290rd16rd16 genotype and in the Cep290rd16/rdl6; $M k k s^{k o / k o}$ genotype (Supplemental Figure 6A). Note the variability in the Cep290rd16/rd16; $M k k s^{k o / k o}$ genotype (Supplemental Figure 6B), though short OSs develop (Supplemental Figure 6C) despite the abnormalities in connecting cilium biogenesis (Figure 5, A-C, and Supplemental Figure 3).

\section{Discussion}

CEP290 is a highly conserved multifunctional protein of $290 \mathrm{kDa}$ (54). Elegant studies in Cblamydomonas have revealed that CEP290 is localized in membrane-microtubule connections at the ciliary transition zone and likely controls the composition of flagella proteins $(34,35)$, consistent with its suggested role in ciliary transport (41). CEP290 is reported to interact with many centriolar and ciliary proteins, such as RPGR (41), CP110 (32), NPHP5 $(20,55)$, and PCM1 $(32,41,53,55$, 56). CEP290 is also required for the localization of small GTPase Rab8 to centrosome and cilia $(32,53)$, thereby playing a critical role in cilia biogenesis. In contrast, MKKS is a chaperonin-related protein in the pericentriolar region and basal body (57), forming complexes with BBS10 and BBS12 (17). Based on the central position of CEP290 at the base of the ciliary transition zone, we hypothesize interactions between CEP290 and other BBS-related proteins. Indeed, our results suggest that structural association and functional linkage of ciliary transport complexes, mediated by CEP290-MKKS interactions, are critical for biogenesis and maintenance of cilia integrity/function.

Human LCA-causing CEP290 mutations are suggested to be hypomorphic (58). Likewise, Cep290rd16 is predicted to be a partially functional mouse allele that retains sufficient CEP290 function to produce cilia when MKKS protein is present; nonetheless, these cilia are functionally abnormal in part due to disrupted anterograde or retrograde transport that results in rapid death of photoreceptors and olfactory dysfunction $(41,42)$. In contrast, $M k k s^{k 0 / k o}$, a null allele, had severely abnormal cilia in all 3 sensory tissues (Figure 5, B and C; Figure 6, A-C and E; and Supplemental Figure 3), suggesting an important role of MKKS in cilia biogenesis; yet, photoreceptor degeneration in this mutant is relatively slow (48). 
A Cep290 dit6rd16

Cep290 dit6rd6; $\quad$ Mkks ${ }^{\text {koko }}$

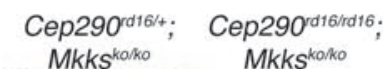

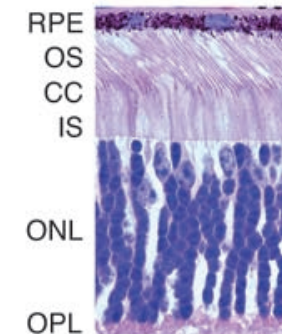

INL $20 \mathrm{O}$

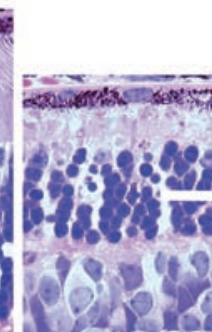

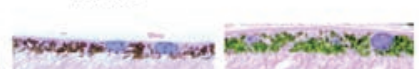

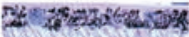

B

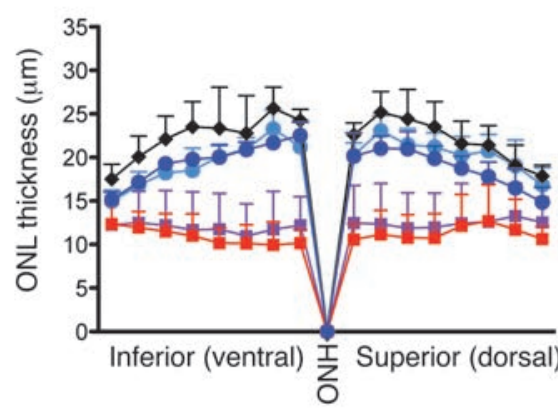

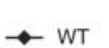

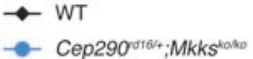

- Mkks

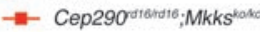

$\rightarrow-$ Cep290 drandir

D

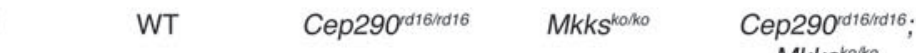

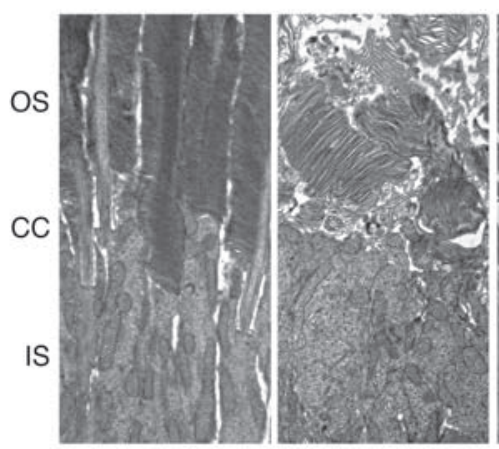

$\mathrm{Mkks}^{\mathrm{k} o / k o}$

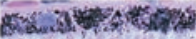

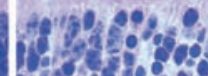
piogis 15 D.

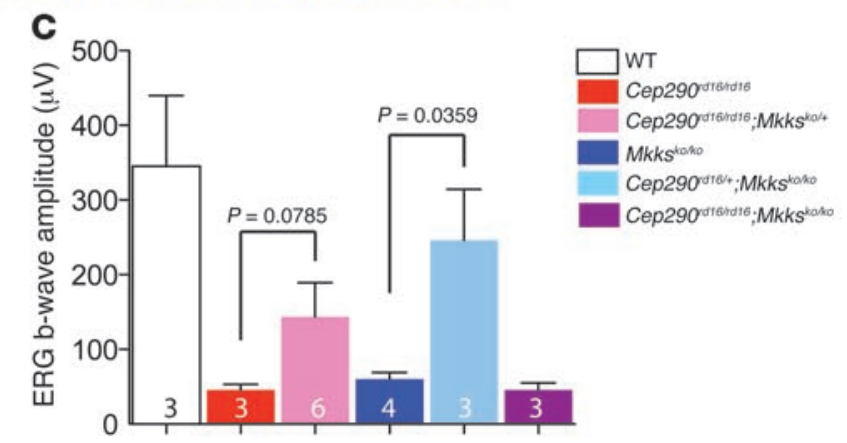

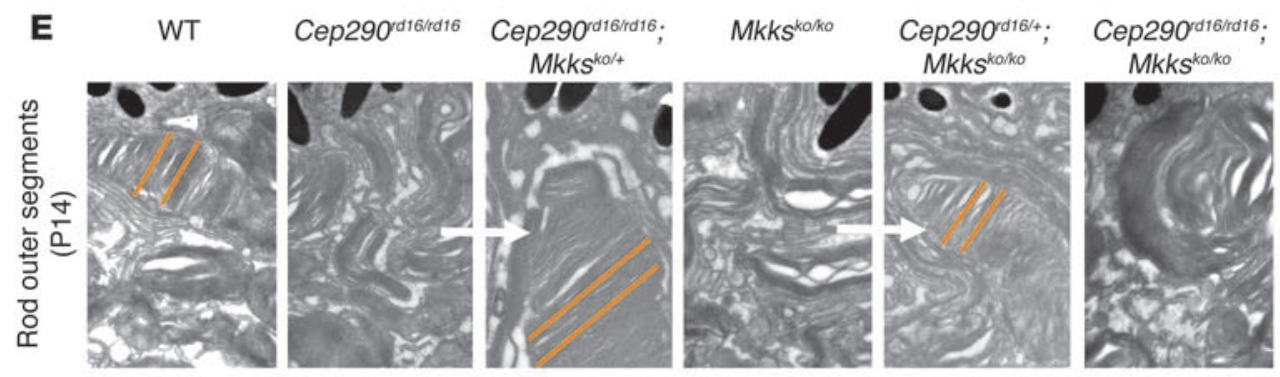




\section{Figure 4}

Triallelic loss of Mkks and/or the Cep290-DSD domain ameliorate cilia phenotypes in photoreceptors. (A) Cross sections through the P18 retina in different combinations of Cep290 ${ }^{\text {rd16 }}$ and $M k k^{k o}$ alleles, as indicated. Note the short, abnormal OSs in Cep290rd16/rd16 or Mkksko/ko genotypes and the more normal OS in the triallelic Cep290 rd16/+;Mkksko/ko genotype. Here, the Cep290 rd16/rd16; $M$ kks $^{k o / k o}$ genotype looks similar to Cep290rd16/rd16. The white arrows indicate comparison between 2 similar genotypes that are improved by combining alleles of Cep290 and Mkks. Original magnification, $\times 40$. (B) Quantitation of outer nuclear layer thickness at P18 in the genotypes indicated. Higher variability is noted in double-homozygous mutants (see error bars on Cep290rd16/rd16 versus Cep290'rd16/rd16; $\mathrm{kkks}^{\mathrm{ko} / \mathrm{ko}}$ ). Error bars are SD; $n=6$ (WT), $n=4$ (Cep290 rd16/+;Mkks $\left.{ }^{k o / k o}\right), n=3$ (Mkks $\left.{ }^{k o / k o}\right), n=14$ (Cep290 rd16/rd16; $\left.M k k s^{k o / k o}\right), n=8$ (Cep290 rd16/rd16). (C) Scotopic ERG b-wave amplitudes in the indicated mouse genotypes (at P20). Removing one WT Mkks allele on a Cep290rd16/rd16 background results in improved responses, as does adding one Cep290rd16 allele on a Mkkskolko background. Note that single homozygous or double-homozygous genotypes have essentially no ERG b-wave response. Error bars are SD; $n=3$ (WT), $n=3\left(\right.$ Cep290 $\left.{ }^{\text {rd16/rd16 }}\right), n=6\left(\right.$ Cep290 $\left.{ }^{\text {rd16/rd16; }} M_{k k s^{k o /}+}\right), n=4\left(M_{k k s k o l k o}\right)$, $n=3$ (Cep290 rd16/+; $\left.M k_{k k s}{ }^{k o / k o}\right)$, and $n=3$ (Cep290rd16/rd16;Mkkskolko). (D) Longitudinal EM sections through the OS, connecting cilia, and inner segments in $\mathrm{P} 14$ retina show that OS morphology is disrupted in the indicated mutant genotypes. Original magnification, $\times 3,000$. (E) Highermagnification images (original magnification, $\times 30,000$ ) of OSs in P14 retina confirm improved OS morphology in Cep290 ${ }^{\text {rd16/+; }} M M_{k} k^{k o / k o}$ and Cep290 ${ }^{\text {rd16/rd16; }}$ Mkks ${ }^{\mathrm{ko} /+}$ triallelic genotypes. OSs of triallelic mice form concentric stacks of discs (parallel orange lines), more similar to WT. The white arrows indicate comparison between 2 similar genotypes that are improved by combining alleles of Cep290 and Mkks. RPE, retinal pigment epithelium; CC, connecting cilia; IS, inner segment; ONL, outer nuclear layer; OPL, outer plexiform layer; INL, inner nuclear layer; $\mathrm{ONH}$, optic nerve head.

A less severe phenotype or even rescue of the defects observed in sensory cells of Cep290rd16 and Mkksko double-homozygous and triple allelic combinations suggest that the CEP290- $\triangle$ DSD protein (produced by the $r d 16$ allele) exhibits novel function(s) to our knowledge in the absence of MKKS. In parallel, we predict that null Cep290 allele(s) in combination with $M k k s^{k o}$ alleles would lead to more severe ciliary defects, as suggested by combined morpholino knockdown data in zebrafish. Together with human Cep290 mutational analysis (54), our results suggest that other hypomorphic alleles of Cep290 may also rescue the sensory phenotype caused by the loss of MKKS.

Our studies show a direct physical and functional link between the DSD domain of CEP290 (in the ciliary transition zone) and MKKS (in the basal body). Disrupting this association appears to be a likely cause of cilia dysfunction and/or cell death in Cep290 rd16/rd16 and $M k k s^{k o / k o}$ mice. Recent studies in Chlamydomonas have implicated a major function for CEP290 as a gatekeeper, regulating protein trafficking at the base of the cilium $(34,35)$. Consistent with such a role, cilia in Cep290 mutants had abnormal accumulations and reductions of various IFT and BBS proteins (34). Interactions between IFT and BBS proteins have been identified in C. elegans (59) and zebrafish retina (60). OSs continuously elongate as the outermost disks are engulfed by the RPE, explaining the importance of the ciliary axoneme to constantly supply new disk components. In Cep290rd16, $M k k s^{k o}$, and combined mutants the axoneme is missing (Figure 4D). The lack of basal body and connecting cilia in double-mutant mice, suggests that MKKS and Cep290 are required for the elaboration of the cilium and for polar segregation of cellular domains.
The DSD domain of Cep290, missing in Cep290rd16, may be essential in sensory neurons, which rely heavily on transport via the cilium (61). Whereas the rd16 mutation results in rapid photoreceptor loss, $M k k s^{k o}$ mice have more severe disruption of OS morphology and slower photoreceptor cell loss. These results are similar to zebrafish IFT mutants, in which photoreceptors fail to develop OS. The IFT57 mutant resembles $r d 16$, in that short OSs develop initially (62), whereas in the IFT88 and IFT172 mutants, disorganized disk membranes accumulate (63), as we observed in the $M k k s^{k 0}$ mice. IFT-A and IFT-B particles have distinct roles in cilia (59). In Chlamydomonas, BBS proteins export signaling proteins through the flagella as IFT cargo (64), and Cep290 mutants have increased IFT proteins and BBS4 (34). These findings suggest complementary roles for different IFT proteins, and likewise for Cep290 and MKKS, in OS maintenance versus formation of the cilium. Although no direct connection among Cep290, BBS, and IFT in mammalian photoreceptors or hair cells has yet been identified, we hypothesize that such a link may be important in sensory neuron function. In mammalian sensory cells, imbalances in regulation of IFT caused by the loss of CEP290-DSD domain may be corrected by the absence of MKKS. Likewise, the severity of ciliary dysfunction was reduced when the DSD domain was removed by introducing Cep290rd16 allele(s) in the $M k k s^{k o / k o}$ genotype (Figure 7F). A link between CEP290 and proteins of the BBS and IFT families can be tested in future experiments by exploring the expression of IFT proteins in these mutants and the interaction of IFT components with CEP290 and MKKS.

Consistent with this hypothesis, recent morphological and functional analysis in C. elegans (18) and mammalian cell lines (20) demonstrated a cooperative role of MKS and NPHP proteins that localize to the transition zone distal to the basal body in creating a gateway for entry to the cilium $(18,20)$. CEP290 has multiple domains and proposed gatekeeping functions that regulate trafficking of an assortment of cargo, which together may explain a large degree of genetic and clinical heterogeneity associated with mutations in this protein. Our identification of an interaction between CEP290-DSD and MKKS provides further mechanistic insight into the roles of these 2 proteins at structural and functional levels.

Improved cilia integrity and biogenesis in combined Cep290-Mkks mutants are particularly intriguing, though sensory neurons in distinct tissues appear to respond differently. Hair cells and olfactory sensory neurons show improvement in both triple mutant allelic combinations and in double-homozygous states. Photoreceptors, however, exhibit a partial rescue of defects only in triple allelic combinations (Cep290 ${ }^{r d 16 /+} ; M k k s^{k o / k o}$ and Cep290 rd16/rd16; $M k k s^{k 0 /+}$ ) but not in the double homozygotes (Cep290rd16/rd16; Mkks $\left.s^{k o / k o}\right)$. This differential response indicates a more fundamental structural and/or functional requirement of CEP290-MKKS interaction in photoreceptor cilia compared with that in cochlear hair cells or olfactory sensory neurons. The photoreceptors have a single long-lasting cilium with immense transport requirements for daily replacement of OS discs (52), whereas cochlear hair cells contain a transient kinocilium required for the establishment of the stereocilia bundles (50). Once a hair cell has reached maturity the kinocilium retracts as it is no longer required. The olfactory sensory neurons display multiple basal bodies and cilia per cell, thus potentially mitigating cilia defects in individual cells (65).

We believe that a unique finding of our study is the demonstration of a physical linkage between proximal cilia (e.g., CEP290) and basal body (e.g., MKKS) proteins. Thus, variants in one 
A

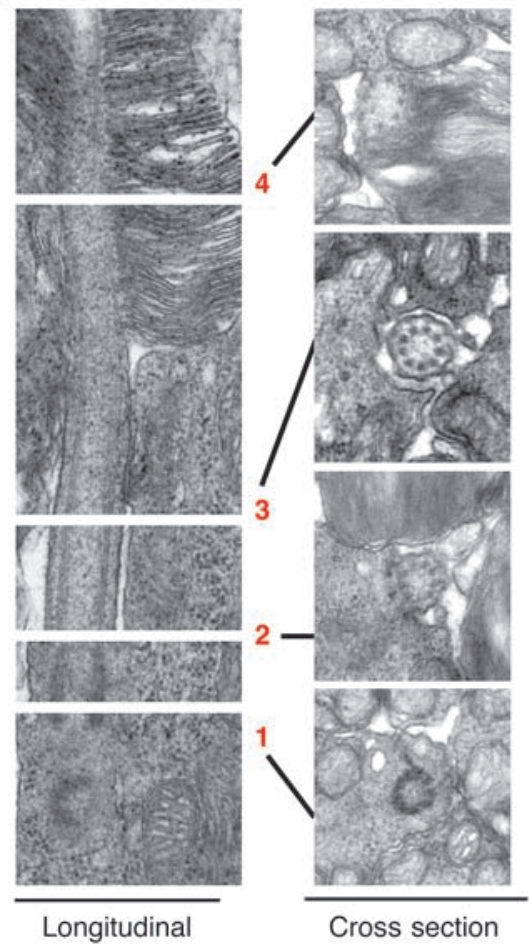

B

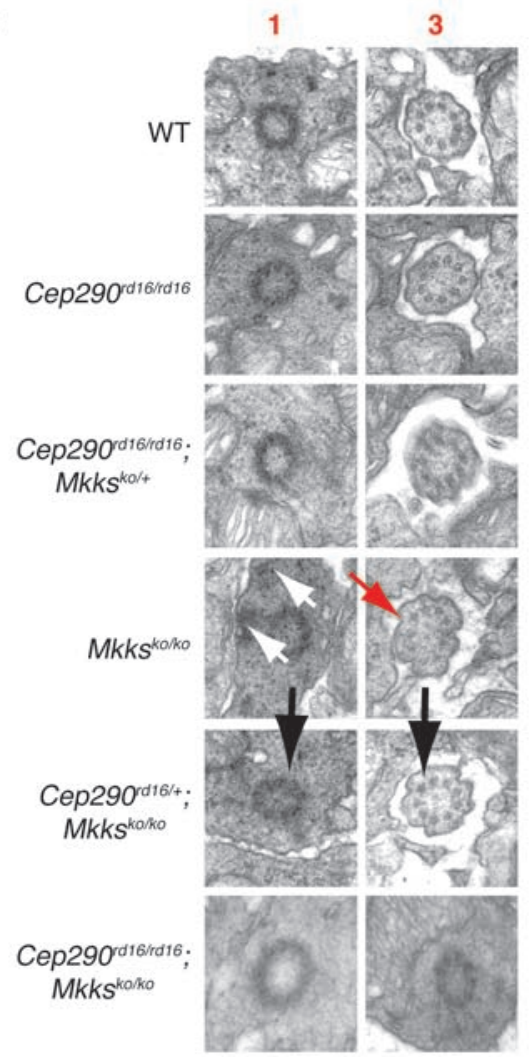

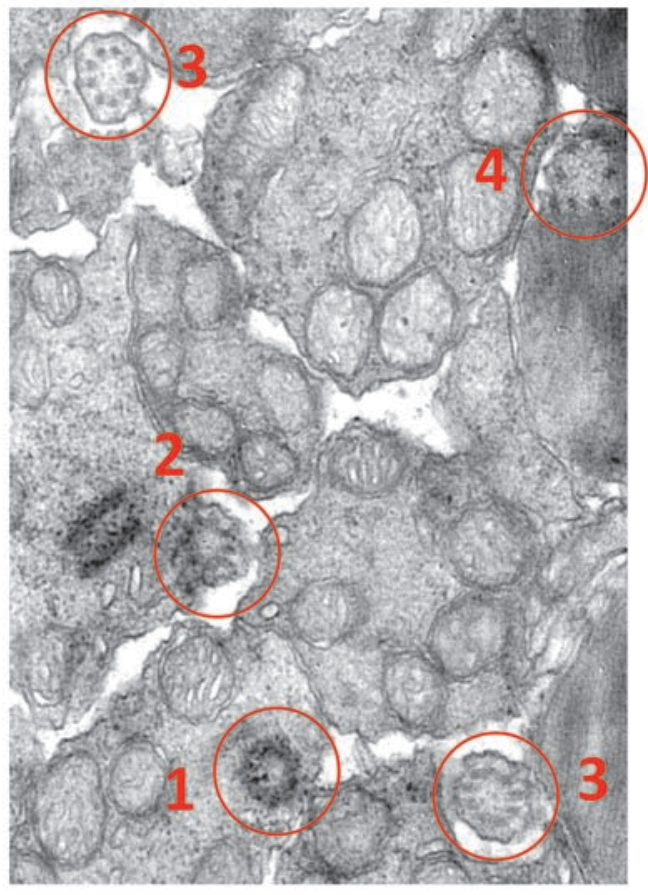

C

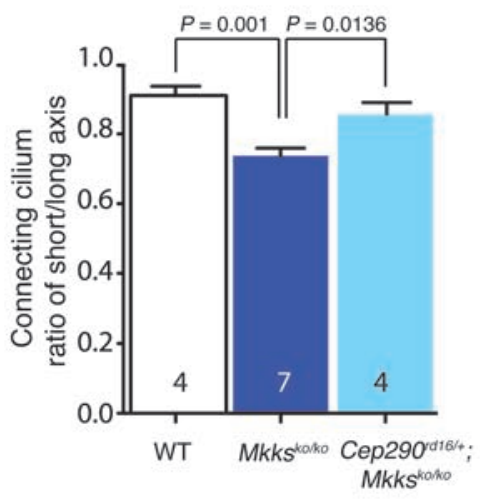

\section{Figure 5}

Ultrastructural (EM) analysis of cilia and basal bodies in P14 WT mouse retinal photoreceptors. (A) Ultrastructural (EM) analysis of cilia and basal bodies in $\mathrm{P} 14$ WT mouse retinal photoreceptors. The left panel shows a longitudinal section electron micrograph, with white lines indicating the plane of the cross section electron micrographs (middle panel) through, 1, the basal body; 2 , transition zone; 3 , connecting cilium; and 4, axoneme of OSs. The right panel shows a representative cross section through inner/OS junction and illustrates each structure in situ. Original magnification, $\times 20,000$. (B) Basal bodies, 1, and connecting cilia, 3 , in cross section, showing the normal $9+0$ arrangement of the microtubule bundles in P14 WT and Cep290rd16/rd16 animals. In $M k k s^{k o / k o}$ animals, note the diffuse pericentriolar material surrounding the basal body (white arrows) and flattened cilium (red arrow). Cep290 rd16/+; $M k k s^{k o / k o}$ mice have improved ciliary and basal body morphology compared with that of single homozygotes. Black arrows indicate comparison between $M k_{k} \mathrm{~s}^{\mathrm{ko} / \mathrm{ko}}$ and Cep290 ${ }^{\text {rd16/+; }: M k k s^{k o / k o} \text { geno- }}$ types. Additional examples of cilia and basal bodies for each genotype are shown in Supplemental Figure 3. Original magnification, $\times 50,000$. (C) Quantitation of connecting cilium short/long diameter ratio in WT, Mkksko/ko, and Cep290rd16/+; $M k k s^{k o l k o}$ genotypes, showing rescue of cilia cross-sectional shape in the triallelic genotype. Original magnification, $\times 50,000$. Error bars are SEM; $n=4$ (WT), $n=7$ $\left(M_{k k s^{k o / k o}}\right)$, and $n=4$ (Cep290 ${ }^{\text {rd16/+*; }}$ $M k k s^{k o l k o}$ ).

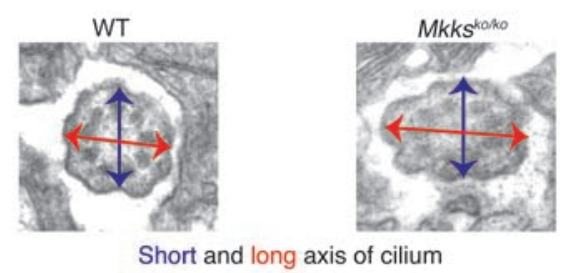

protein may impact the structure and/or function of the entire cilium. The coexisting alterations in an interacting protein(s) can attenuate (as reported here) or deteriorate (as in the case of RPGRIP1L, ref. 13, and AHI1, refs. 13, 14) ciliopathy phenotypes. A range of phenotypic variability in the Cep290rd16/rd16; $M k k s^{k o / k o}$ genotype is consistent with the hypothesis that individual cells vary in their ability to compensate for disruptions in ciliary complexes. Modest changes in protein interactions can modify these disruptions and lead to cellular pathology or death, analogous to the "butterfly effect" in chaos theory, in which tiny wing move- 
A

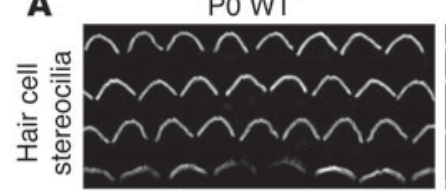

B
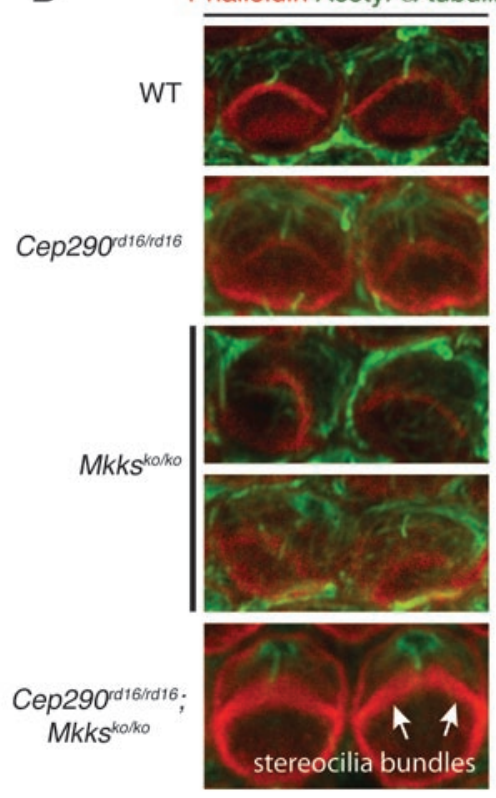

E WT

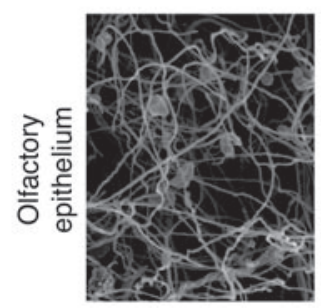

Cep290'di6/16

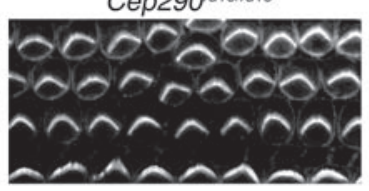

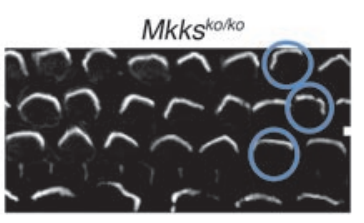

Cep290 ditirdr $;$ Mkks kolko

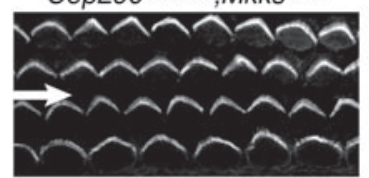

C
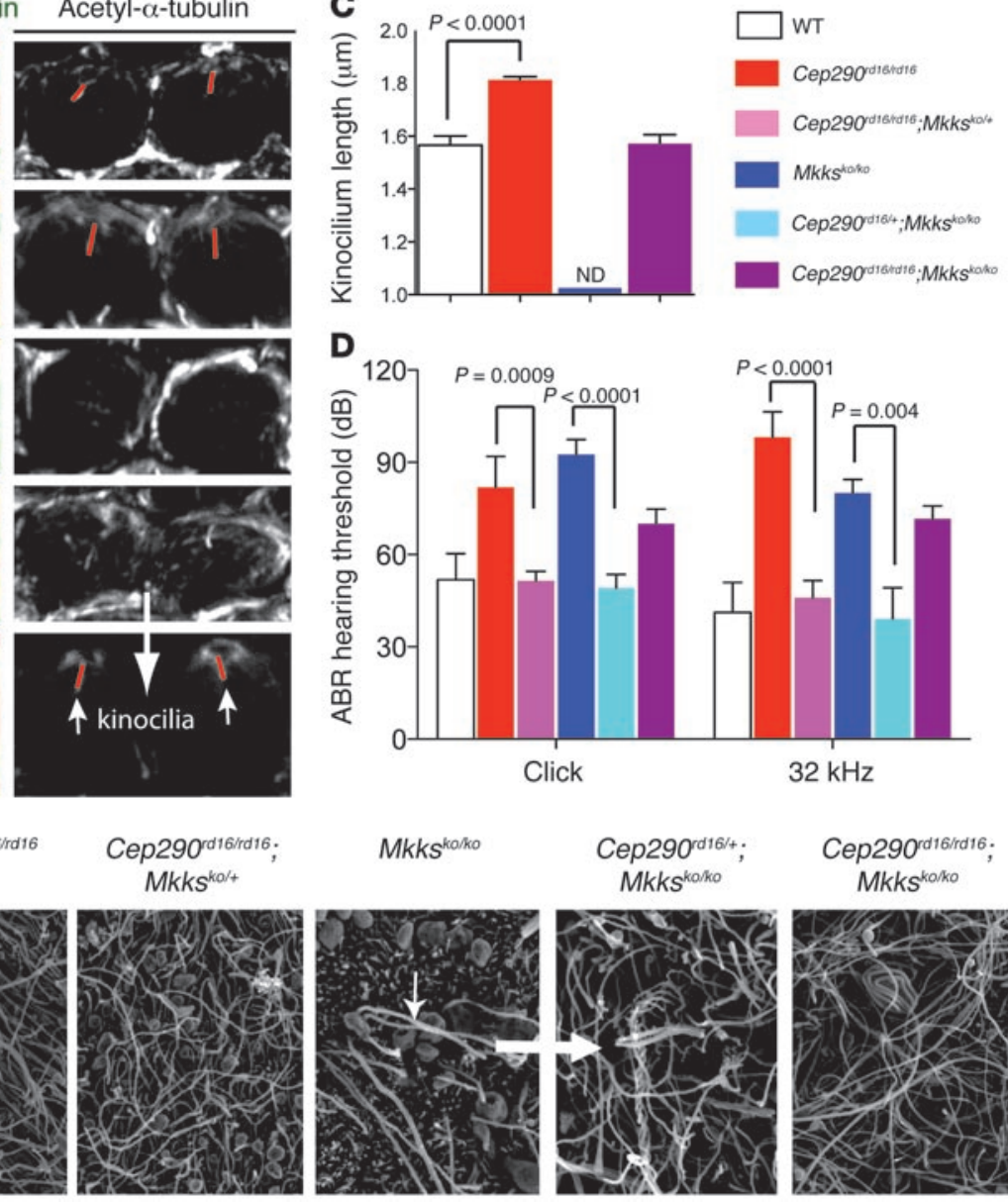

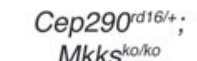
Mkks kakko

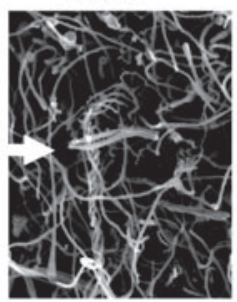

Cep290'd16rd16; $\mathrm{Mkks}^{\text {kolko }}$

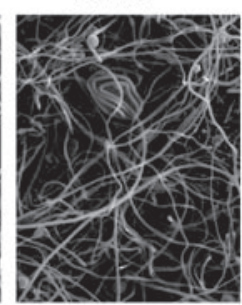

\section{Figure 6}

Loss of MKKS in combination with CEP290-DSD rescues hair cell kinocilia and olfactory sensory cilia defects. (A) Confocal images of stereociliary bundles of hair cells from P0 mice. Minor defects in stereociliary bundle architecture in Cep290rd16/rd16 mice compared with those in P0 WT mice and marked defects in bundle morphology in $M k k s^{k o l k o}$ mice (blue circles). Bundle morphology is normal in double mutants (white arrow). Original magnification, $\times 75.6$. (B) Higher-magnification image (original magnification, $\times 116$ ) of phalloidin-labeled stereocilia bundles (red) and acetyl- $\alpha$-tubulin-marked kinocilia (green) of outer hair cells in P0 cochlea. In the adjacent monochromatic panel, red lines identify kinocilia. Abnormal bundle rotation and misplaced, malformed, or missing kinocilia in Mkkskolko cells are rescued in the double mutant (white arrows). (C) Quantitation of kinocilium length. Cep290rd16/rd16 cilia are significantly longer than WT cilia; Mkkskolko kinocilia are highly abnormal or absent; double-mutant kinocilia are indistinguishable from WT kinocilia. ND, not determined. Error bars are SD; kinocilia measured, respectively, are $n=42$ (WT), $n=77$ (Cep290 rd16/rd16), and $n=35$ (Cep290 rd16/rd16; Mkkskolko). (D) ABRs in 3- to 4-month-old mice of indicated genotypes, with mean threshold \pm SD. Mkkskolko and Cep290 rd16/rd16 mice show elevated hearing thresholds. Complete rescue of ABR thresholds in animals with triallelic combinations and partial rescue in double homozygotes. Error bars are SD; cochlea examined for each condition, respectively, are $n=8$ (WT),

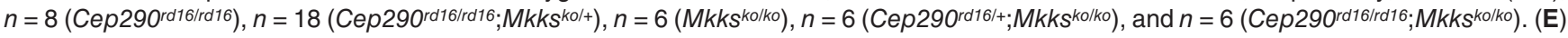
SEM images of olfactory epithelium at 7 to 8 weeks of age, showing loss of cilia in Mkkskolko mice (small white arrow) and their retention in the double-mutant and triple allelic combinations (large white arrow). Original magnification, $\times 6,500$.

ments in a sensitive dynamic system ultimately have far-reaching effects (66). In concordance, extensive phenotypic variations are reported in patients with LCA and BBS $(12,54)$. In addition to restoring the missing or abnormal protein, our studies suggest a therapeutic approach for treating ciliopathies by targeting one or more interacting proteins.

\section{Methods}

Patients and mutation screening. LCA is defined by poor fixation and wandering nystagmus with congenital onset before 6 months of age, amaurotic pupils, absent or severely decreased ERG before age 12 months, and essentially normal retinal appearance with no systemic disease. Patients with juvenile-onset retinitis pigmentosa (RP) have decreased vision, with 
A CEP290 Rootletin MKKS Rootletin
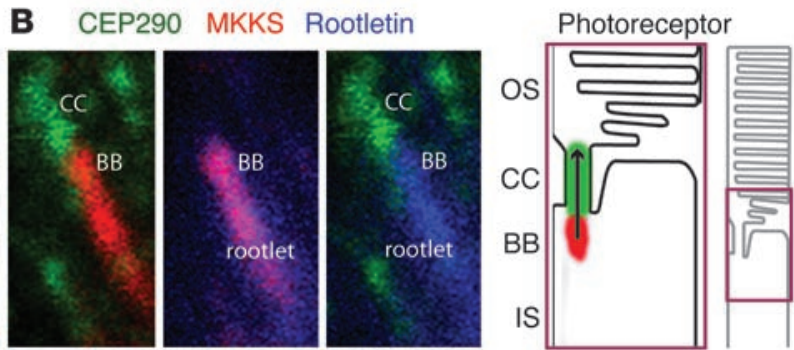

C
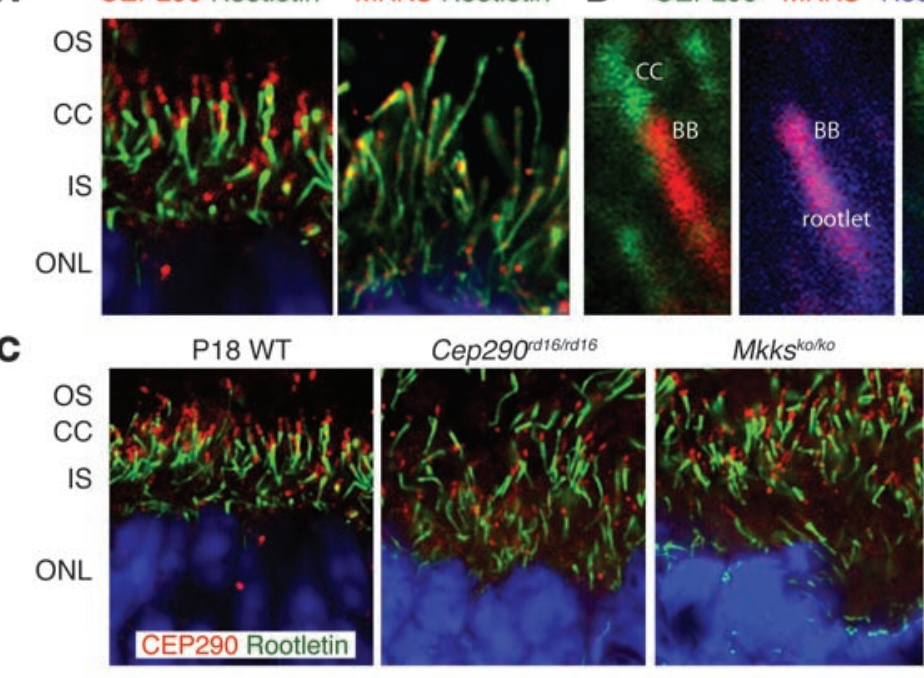

Cep290 ${ }^{\text {rd16rd16 }} ; M_{k k s^{k o / k o}}$

D

PO WT CEP290 $\gamma$-Tubulin
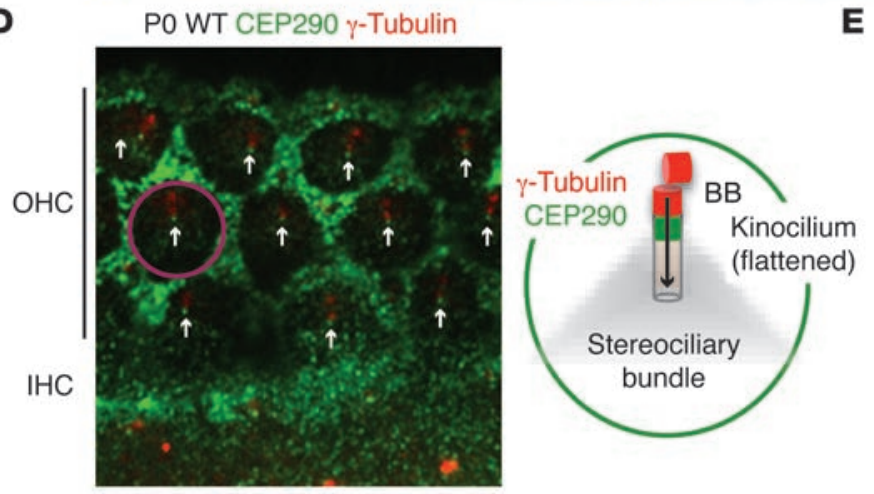

E CEP290 $\gamma$-Tubulin
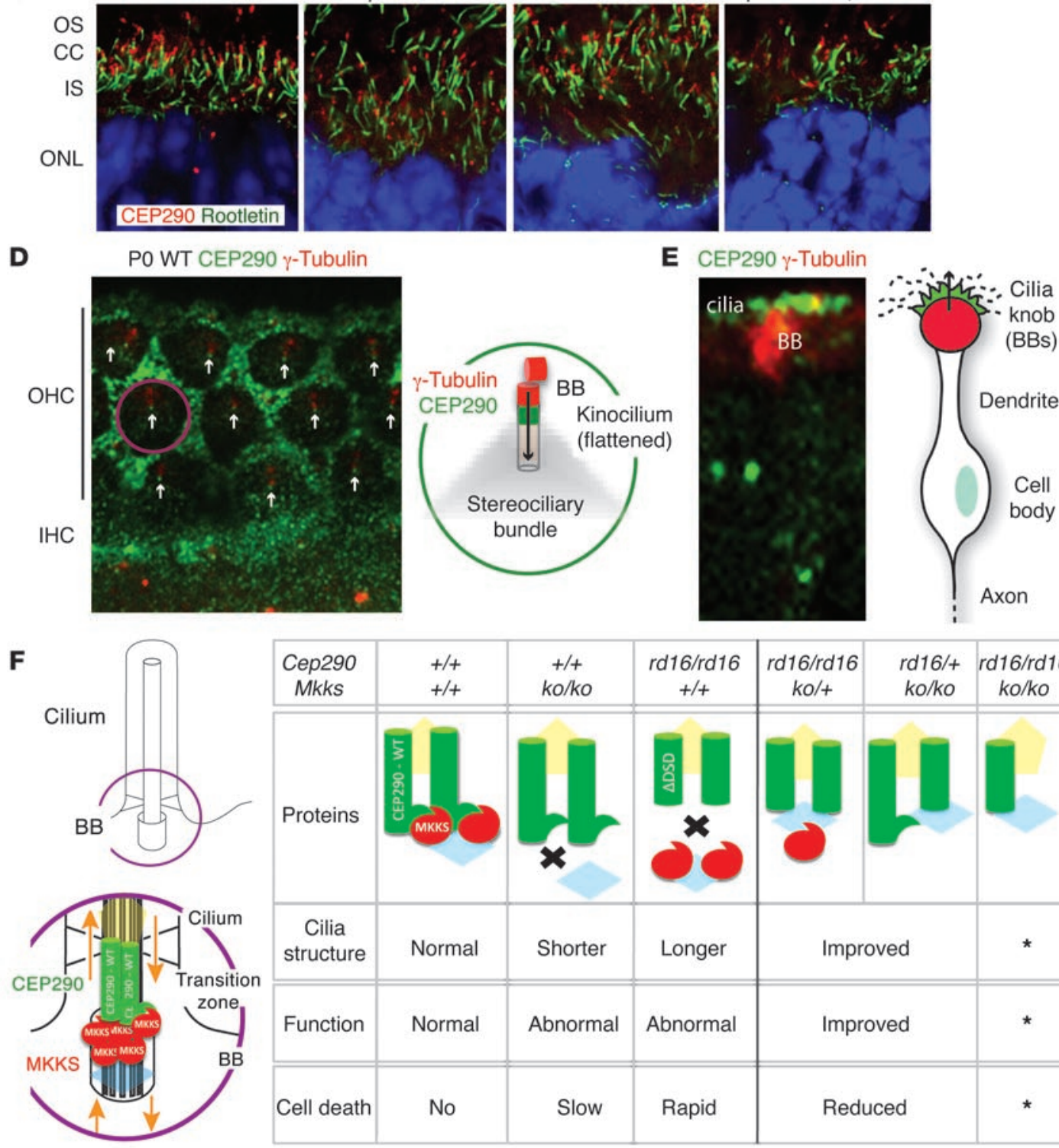

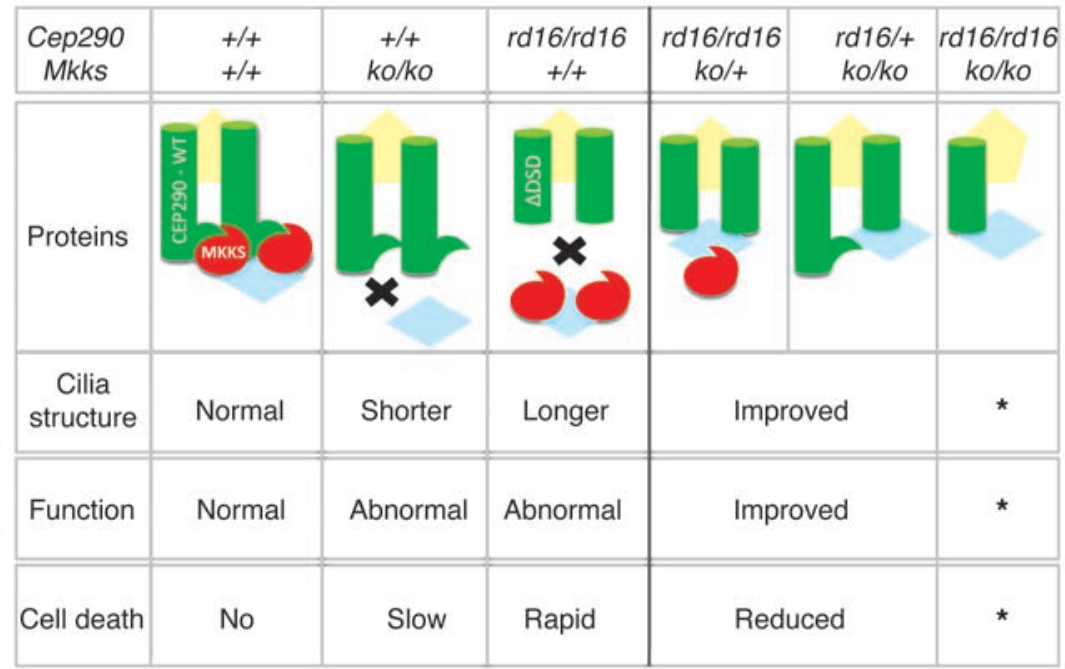




\section{Figure 7}

CEP290 and MKKS are expressed in adjacent domains in ciliated sensory cells. (A) CEP290 (red) and rootletin (green) in WT photoreceptors. CEP290 localizes to the connecting cilium, distal to ciliary rootlet. MKKS (red) and rootletin (green); MKKS caps the rootlet in the basal body (BB) region. Original magnification, $\times 180$. (B) CEP290 (green), MKKS (red), and rootletin (rootlet; blue) in photoreceptors. CEP290 and MKKS are expressed in adjacent, nonoverlapping domains corresponding to connecting cilium and basal body. Arrow shows proximaldistal orientation of cilium. Original magnification, $\times 480$. (C) CEP290 (red) continues to be expressed in Cep290rd16/rd16, Mkkskolko, and in double homozygotes, albeit irregularly and disorderly. Original magnification, $\times 180$. (D) Expression of CEP290 (green) and $\gamma$-tubulin (red) in PO WT cochlea. In hair cells, CEP290 is detected in punctate spots at base of kinocilium (white arrows); $\gamma$-tubulin (red) localizes to the basal body. CEP290 is expressed broadly in supporting cells surrounding hair cells. Diagram shows enlargement of the hair cell (purple circle). $\mathrm{IHC}$, inner hair cells; OHC, outer hair cells. Original magnification, $\times 176.4$. (E) CEP290 (green) and $\gamma$-tubulin (red) expression in olfactory sensory neurons on the surface of the olfactory epithelium. Olfactory sensory neurons have multiple basal bodies and cilia per cell (schematic on right), with arrow indicating proximal-distal direction. Original magnification, $\times 60$. (F) A possible model depicting interaction of CEP290-DSD in proximal cilia, with MKKS in adjacent basal bodies. The long green cylinder indicates CEP290, with the hook indicating the MKKS-interacting DSD domain. The short green cylinder represents CEP290- $\triangle$ DSD, and the red oval represents MKKS. The yellow pentagon and blue diamond represent predicted CEP290- and MKKS-interacting proteins, respectively. Asterisks indicate that the effect of losing both alleles is tissue specific. In the cochlea and olfactory epithelium, improvement is noted with loss of both alleles. In photoreceptors, such improvement does not occur.

or without nystagmus (noted later at around 1 year age) and with nyctalopia and an abnormal, diminished ERG at around 1 year age. We excluded systemic disease by history and pediatric examinations.

DNA was extracted with a QIAamp DNA Blood Kit (Qiagen) from peripheral blood according to standard procedures. A total of 142 DNA samples from patients with LCA or juvenile RP were sequenced for variants in the $M K K S$ gene (NCBI accession no. NM170784). Of these, 58 patients had potentially disease-causing CEP290 sequence changes, and 84 patients were negative for the common $(\sim 500)$ mutations in 12 reported LCA genes tested by APEX technology (http://www.asperbio.com/asper-ophthalmics) or Carver laboratory technology (https://www.carverlab.org/). PCR amplification was performed with intronic primers designed to flank the splice junctions of each coding amplicon of the MKKS gene (see Supplemental Table 1 for primer sequences). PCR products were subjected to automated DNA sequencing using BigDye Terminator Cycle Sequencing Kit (Applied Biosystems) and a capillary sequencer (Applied Biosystems no. 3730XL2). Sequences were analyzed using SeqMan (DNASTAR).

$M K K S$ variants that were present in the SNP database (NCBI) were exclud$\mathrm{ed}$, and the remaining variants were examined in at least 100 ethnically matched control subjects and against the 1,000 Genome database (http:// browser.1000genomes.org/index.html). Cosegregation analysis of variants was performed when families were available. We carried out in co analyses, including Blosum62, SIFT, Polyphen (NCBI), and conservation comparisons, to predict the impact of the MKKS variant on protein function. Clinical history and genetic characteristics of patients with potentially diseasecausing $M K K S$ variants are included in the Supplemental Methods.

Zebrafish morpholinos. Morpholinos, tagged with fluorescein (Gene Tools Inc.) and targeted against translation-initiating AUG codons, have the following sequences: $M k k s, 5^{\prime}$-TTCTTCTTACTAATGCGAGACATGC-3' (44), and Cep290, 5'-GCCGCAGGCATTCTTCAGGTCAGCT-3'
(27). A standard negative control morpholino and translational blocking morpholinos against zebrafish genes Cep290 (27) and Mkks (43) were quantified spectrophotometrically (NanoDrop Tech Inc.). Staged Danio rerio embryos, between 2 to 8 cells, were microinjected with morpholino $(0.4-1.2 \mathrm{nl})$ into the yolk sac. Each needle was calibrated against a micrometer to determine the volume delivered per pulse. Microinjected embryos were incubated at $28^{\circ} \mathrm{C}$ overnight and scored for survival the following day. At 72 hours after fertilization, live embryos with fluorescein signal were observed and recorded (Leica), after which they were fixed with $4 \%$ glutaraldehyde at room temperature for 30 minutes and then in $4 \%$ paraformaldehyde overnight at $4^{\circ} \mathrm{C}$. Fixed tissues were washed with PBS and embedded in OCT compound (Tissue-Tek). Sections $(10 \mu \mathrm{m})$ were stained with standard $\mathrm{H} \& \mathrm{E}$ protocol.

Animals and functional testing of mice. Cep290rd16/rd16 mutant mice (41) on a C57BL/6J background and Mkks $s^{k / k o}$ mice $(48,49)$ on a mixed 129/Sv and C57BL/ 6 background were used to generate double mutants on a mixed C57BL/6 and 129 background (approximately 75\% C57BL/6). P0 is considered the day of birth. After euthanasia by carbon dioxide inhalation, eyes were collected and immersion fixed in 4\% PFA for 4 hours followed by cryoprotection in graded sucrose, frozen, and sectioned at $10 \mu \mathrm{m}$. For some experiments, the eyes were fixed in $2 \%$ glutaraldehyde for 30 minutes, transferred to $10 \%$ buffered formalin or $4 \%$ PFA until processing, embedded in methacrylate, sectioned, and stained with H\&E or PAS.

Measurement ofhearingthreshold/ABR. Mice were divided into 6 groups depending upon their genotype: control (WT, Mkks $s^{k o /+}$, or Cep290 $\left.0^{r d 16 /+}\right)(n=8$ ears), Cep290 ${ }^{\text {rd16/rd16 }}\left(n=8\right.$ ears), Mkksko/ko ( $n=6$ ears), Cep290rd16/+; $M k k s^{k o / k o}(n=6$ ears), Cep290 ${ }^{\text {rd16/rd16 }} ; M_{k k s^{k o /+}}\left(n=18\right.$ ears), and Cep290 rd16/rd16; $M k k s^{k 0 / k o}(n=6$ ears). ABR recordings were made in a sound-attenuated booth using an auditory-evoked potential system with high-frequency transducers (Intelligent Hearing Systems). Waveform acquisitions were generated by averaging the response from 1,024 stimuli. Stimuli were presented at $21.1 \mathrm{~Hz}$ with alternating polarity. The maximum sound intensity tested for click and tone-burst stimulus was $120 \mathrm{~dB}$ SPL, except for $32 \mathrm{kHz}$ tone-burst stimuli where $100 \mathrm{~dB}$ of maximum sound intensity was used. The intensity of stimuli was gradually decreased by $5 \mathrm{~dB}$ until reproducibility of waveforms was lost. A threshold was defined as the lowest sound intensity that can reproduce waveforms. ABR thresholds from each genotype were averaged and compared among the groups. Since ABR thresholds achieved from the click and 8-kHz stimuli did not follow a normal distribution, Mann-Whitney test was used for comparing the average of those values. Otherwise, $t$ test was used for comparisons.

Funduscopy. Mice were anesthetized with ketamine and xylazine, and pupils were dilated with topical $0.5 \%$ tropicamide and $1 \%$ cyclopentolate hydrochloride. The endoscope contacted the corneal surface through a layer of $1 \%$ GSP. Using a fundus imaging system 16 , we connected a $5-\mathrm{cm}$ long tele-otoscope (1218AA; Karl Storz) and digital camera (D80; Nikon) with an additional +5.00 magnifying lens. The setting of the camera was as follows: large image; operating mode P, and white balance set at Shade. A Xenon lamp (201315-20; Karl Storz) connected through an optic fiber to the tele-otoscope was used as the light source.

Antibodies. CEP290 rabbit polyclonal antibody has been described previously (41). Another Cep290 antibody (Primm Biotech) was made by immunizing 3 rats with an E. coli-expressed recombinant protein carrying a His-tagged CEP290 domain (amino acids 2217-2396). The antiserum was characterized by ELISA and purified using an affinity column made by cross-linking the recombinant CEP290 protein domain. The antibody (CP290PB_Rat) was diluted (50\% glycerol) and stored in aliquots at a concentration of $1 \mathrm{mg} / \mathrm{ml}$. The purified antibodies were tested in immunoblots of mouse retina extracts and detected a major band of $290 \mathrm{kDa}$. Commercial antibodies included anti-BBS6 (Abnova), anti-acetylated $\alpha$-tubulin 
and anti- $\gamma$ tubulin mouse monoclonals (Sigma-Aldrich), and anti-rootletin (67). Secondary antibodies included Alexa Fluor 488, Alexa Fluor 546, Alexa Fluor 568, and phalloidin (Molecular Probes).

Confocal microscopy. Cryosections $(10 \mu \mathrm{m})$ were collected and immunolabeled with antibodies. After overnight incubation in primary antibody, sections were washed and incubated in Alexa Fluor-conjugated goat antimouse and goat anti-rabbit secondary antibody (Invitrogen). DAPI was included in incubation buffer to label nuclei. Sections were washed and transferred to glass slides for imaging. Primary antibodies were omitted from samples prepared as negative controls. Samples were imaged on either a Leica SP2 or Olympus FV1000 laser scanning confocal microscope using a $\times 63$ oil immersion objective. Samples were imaged in sequential scan mode to minimize bleed through and exported as noncompressed TIFF files.

$E M$. Mice were deeply anesthetized with ketamine $(50 \mathrm{mg} / \mathrm{kg})$ and xylazine $(5 \mathrm{mg} / \mathrm{kg})$ and perfused transcardially with $2 \% \mathrm{PFA} / 2.5 \%$ glutaraldehyde in $0.1 \mathrm{M}$ phosphate buffer ( $\mathrm{pH}$ 7.4). Eyes were enucleated and hemisected, and the posterior eyecup segments were immersed in fixative for 2 hours and rinsed in PBS at $4{ }^{\circ} \mathrm{C}$ overnight. For transmission EM, the fixed eyecups were dehydrated in ethanol in series $(50 \%, 70 \%$, and $95 \%)$, block-stained in $1 \%$ uranyl acetate in absolute ethanol for 1 hour, rinsed in $\times 2$ absolute ethanol, and embedded via propylene oxide in epoxy resin (Embed 812; Electron Microscopy Science). Ultrathin sections were cut, post-stained in uranyl acetate and lead citrate, and observed by electron microscope (JEOL1010X, Peabody).

Quantitation. To quantify kinocilia length, cochlear whole mounts were imaged using a Zeiss confocal microscope. Only cilia lying flat within a $1.5-\mu \mathrm{m}$ section were measured. Cilia were confirmed to be lying flat by observing a cross-sectional view of the scanned tissue, which included the individual cilia. To quantify photoreceptor cilium cross-sectional diameter ratios, the long and short diameters of connecting cilia EM images were measured in ImageJ (http://rsbweb.nih.gov/ij/), and ratios of short/long axes were calculated.

Yeast 2-hybrid analysis. In other experiments, full-length MKKS-pGBDU and a series of various missense variants (NCBI no. CAC16847.1; p.Y37C, p.G52D, p.T57A, p.T112A, p.S236P, p.A242S, p.L277P, p.D286A, p.T325A, p.G345F, p.C499S) were cotransformed with Cep290-DSD-pGADT7 into PJ69-4A yeast competent cells and plated on -L-U selective medium. Individual cotransformants were streaked onto high stringency selection plates (-U-L-H) and analyzed for growth using 10-fold serial dilution assay.

Mammalian cell culture, transfection, and coimmunoprecipitation assays. HEK293 cells were maintained in Dulbecco's modified Eagle medium (Invitrogen), supplemented with $10 \%$ fetal bovine serum and $1 \mathrm{x}$ penicillin/streptomycin/amphotericin (Sigma-Aldrich), and grown at $37^{\circ} \mathrm{C}$ in $5 \% \mathrm{CO}_{2}$. Cells were grown in 6-well dishes, transfected with Cep290-pGFP and MKKSpCMV myc containing missense variants (NCBI no. CAC16847.1; p.G52D, p.T57A, p.L277P, p.G345E, p.C499S) using Lipofectamine (Invitrogen), and harvested after 48 hours by scraping into $1 \mathrm{ml}$ ice-cold RIPA buffer (Invitrogen) supplemented with protease inhibitors (Roche). Cell lysates were immunoprecipitated overnight at $4^{\circ} \mathrm{C}$ with $1 \mu \mathrm{l}$ rabbit polyclonal anti-EGFP
(Clontech). Immunoprecipitate was collected using Dynabeads Protein G magnetic beads (Invitrogen), per the manufacturer's instructions. Beads were washed 4 times with $1 \mathrm{ml}$ fresh RIPA buffer and resuspended in $1 \times$ SDSPAGE sample buffer. Immuoblotting was carried out using monoclonal anti-myc (Sigma-Aldrich) as probe. Membranes were blocked with 5\% milk, probed with the appropriate antibody, and detected by ECL (Amersham). Coimmunoprecipitation from bovine retinal extracts was performed using anti-MKKS and anti-CEP290 antibodies, as described previously (68).

Statistics. All statistical analyses are presented with Prism (GraphPad software). Two-way comparisons in Figure 4C, Figure 5C, and Figure 6, C and D, used 2-tailed Student's $t$ tests. $P$ values of less than 0.05 were considered significant in all experiments.

Study approval. Human blood samples and pedigrees were collected after obtaining informed consent from patients/families. The consent forms and studies adhered to the tenets of Helsinki and were approved by the institutional review boards of the University of Pennsylvania and the McGill University Health Centre. Mouse experiments were approved by the Animal Care and Use Committee (ACUC) of the National Eye Institute, conforming to ARVO guidelines. The zebrafish were maintained under a National Human Genome Research Institute ACUC-approved protocol.

\section{Acknowledgments}

We are grateful to Chi-Chao Chan, Robert Fariss, Jun Zhang, Jacob Nellissery, Awais Zia, Milton English, Kevin Bishop, Anastasia Krasnoperova, Rima Kikani, Tiziana Cogliati, Christopher Brinson, and Corey Williams for discussions or assistance. This work was supported by intramural and extramural funds from the National Eye Institute, National Institute on Deafness and Other Communication Disorders, and National Human Genome Research Institute. Additional support was provided by CIHR, FFB-C, FRSQ, Midwest Eye Banks and Transplantation Center, Reseau Vision, and TFFR. P.L. Beales is a Wellcome Trust Senior Research Fellow.

Received for publication September 14, 2011, and accepted in revised form January 18, 2012.

Address correspondence to: Anand Swaroop, Neurobiology Neurodegeneration and Repair Laboratory, National Eye Institute, NIH, Bethesda, Maryland 20892, USA. Phone: 301.905.7212; Fax: 301.480.9917; E-mail: swaroopa@nei.nih.gov.

Byung Yoon Choi's present address is: Department of Otorhinolaryngology, Seoul National University College of Medicine, Seoul National University Bundang Hospital, Seongnam, Republic of Korea.

Hemant Khanna's present address is: Department of Ophthalmology, University of Massachusetts Medical School, Worcester, Massachusetts, USA.
1. Eggenschwiler JT, Anderson KV. Cilia and developmental signaling. Annu Rev Cell Dev Biol. 2007; 23:345-373.

2. Jones $\mathrm{C}$, et al. Ciliary proteins link basal body polarization to planar cell polarity regulation. Nat Genet. 2008;40(1):69-77.

3. Singla V, Reiter JF. The primary cilium as the cell's antenna: signaling at a sensory organelle. Science. 2006;313(5787):629-633.

4. Ishikawa H, Marshall WF. Ciliogenesis: building the cell's antenna. Nat Rev Mol Cell Biol. 2011; 12(4):222-234.

5. Pedersen LB, Geimer S, Rosenbaum JL. Dissecting the molecular mechanisms of intraflagellar transport in chlamydomonas. Curr Biol. 2006; 16(5):450-459.

6. Liu Q, et al. The proteome of the mouse photoreceptor sensory cilium complex. Mol Cell Proteomics. 2007;6(8):1299-1317.

7. Gherman A, Davis EE, Katsanis N. The ciliary proteome database: an integrated community resource for the genetic and functional dissection of cilia. Nat Genet. 2006;38(9):961-962.

8. Badano JL, Mitsuma N, Beales PL, Katsanis N. The ciliopathies: an emerging class of human genetic disorders. Annu Rev Genomics Hum Genet. 2006; 7:125-148.

9. Baker K, Beales PL. Making sense of cilia in disease: the human ciliopathies. Am J Med Genet C Semin Med Genet. 2009;151C(4):281-295.

10. Nigg EA, Raff JW. Centrioles, centrosomes, and cilia in health and disease. Cell. 2009;139:663-678.

11. Goetz SC, Anderson KV. The primary cilium: a signalling centre during vertebrate development. Nat Rev Genet. 2010;11(5):331-344.

12. Waters AM, Beales PL. Bardet-Biedl syndrome. In: Pagon RA, Bird TD, Dolan CR, Stephens K, eds. Gene Reviews. University of Washington, Seattle, Washington, USA; 2010.

13. Khanna H, et al. A common allele in RPGRIP1L is a modifier of retinal degeneration in ciliopathies. Nat Genet. 2009;41(6):739-745. 
14. Louie CM, et al. AHI1 is required for photoreceptor outer segment development and is a modifier for retinal degeneration in nephronophthisis. Nat Genet. 2010;42(2):175-180

15. Nachury MV, et al. A core complex of BBS proteins cooperates with the GTPase Rab8 to promote ciliary membrane biogenesis. Cell. 2007;129(6):1201-1213.

16. Scholey JM. Intraflagellar transport motors in cilia: moving along the cell's antenna. J Cell Biol. 2008; 180(1):23-29.

17. Seo S, et al. BBS6, BBS10, and BBS12 form a complex with CCT/TRiC family chaperonins and mediate BBSome assembly. Proc Natl Acad SciUS A. 2010; 107(4):1488-1493.

18. Williams CL, et al. MKS and NPHP modules cooperate to establish basal body/transition zone membrane associations and ciliary gate function during ciliogenesis. J Cell Biol. 2011;192(6):1023-1041.

19. Ocbina PJ, Eggenschwiler JT, Moskowitz I, Anderson KV. Complex interactions between genes controlling trafficking in primary cilia. Nat Genet. 2011;43(6):547-553.

20. Sang L, et al. Mapping the NPHP-JBTS-MKS protein network reveals ciliopathy disease genes and pathways. Cell. 2011;145(4):513-528.

21. Ezratty EJ, Stokes N, Chai S, Shah AS, Williams SE, Fuchs E. A role for the primary cilium in notch signaling and epidermal differentiation during skin development. Cell. 2011;145(7):1129-1141.

22. Lancaster MA, Schroth J, Gleeson JG. Subcellular spatial regulation of canonical Wnt signalling at the primary cilium. Nat Cell Biol. 2011;13(6):700-707.

23. den Hollander AI, Roepman R, Koenekoop RK, Cremers FP. Leber congenital amaurosis: genes, proteins and disease mechanisms. Prog Retin Eye Res. 2008;27(4):391-419.

24. Wiszniewski W, et al. Potential involvement of more than one locus in trait manifestation for individuals with Leber congenital amaurosis. Hum Genet. 2011;129(3):319-327.

25. Brancati F, et al. CEP290 mutations are frequently identified in the oculo-renal form of Joubert syndrome-related disorders. Am J Hum Genet. 2007; 81(1):104-113.

26. Doherty D. Joubert syndrome: insights into brain development, cilium biology, and complex disease. Semin Pediatr Neurol. 2009;16(3):143-154.

27. Sayer JA, et al. The centrosomal protein nephrocystin- 6 is mutated in Joubert syndrome and activates transcription factor ATF4. Nat Genet. 2006; 38(6):674-681.

28. Baala L, et al. Pleiotropic effects of CEP290 (NPHP6) mutations extend to Meckel syndrome. Am J Hum Genet. 2007;81(1):170-179.

29. Frank V, et al. Mutations of the CEP290 gene encoding a centrosomal protein cause Meckel-Gruber syndrome. Hum Mutat. 2008;29(1):45-52.

30. Helou J, et al. Mutation analysis of NPHP6/ CEP290 in patients with Joubert syndrome and Senior-Loken syndrome. J Med Genet. 2007; 44(10):657-663.

31. Leitch CC, et al. Hypomorphic mutations in syndromic encephalocele genes are associated with Bardet-Biedl syndrome. Nat Genet. 2008; 40(4):443-448.

32. Tsang WY, et al. CP110 suppresses primary cilia formation through its interaction with CEP290, a protein deficient in human ciliary disease. Dev Cell. 2008;15(2):187-197.

33. Murga-Zamalloa CA, et al. Accumulation of the Raf-1 kinase inhibitory protein (Rkip) is asso- ciated with Cep290-mediated photoreceptor degeneration in ciliopathies. J Biol Chem. 2011; 286(32):28276-28286.

34. Craige B, et al. CEP290 tethers flagellar transition zone microtubules to the membrane and regulates flagellar protein content. J Cell Biol. 2010; 190(5):927-940.

35. Betleja E, Cole DG. Ciliary trafficking: CEP290 guards a gated community. Curr Biol. 2010; 20(21):R928-R931.

36. den Hollander AI, et al. Identification of novel mutations in patients with Leber congenital amaurosis and juvenile RP by genome-wide homozygosity mapping with SNP microarrays. Invest Ophthalmol Vis Sci. 2007;48(12):5690-5698.

37. Beales PL, et al. Genetic and mutational analyses of a large multiethnic Bardet-Biedl cohort reveal a minor involvement of BBS6 and delineate the critical intervals of other loci. Am J Hum Genet. 2001; 68(3):606-616.

38. Hichri H, et al. Testing for triallelism: analysis of six BBS genes in a Bardet-Biedl syndrome family cohort. Eur J Hum Genet. 2005;13(5):607-616.

39. Slavotinek AM, et al. Mutations in MKKS cause Bardet-Biedl syndrome. Nat Genet. 2000;26(1):15-16.

40. Stone DL, et al. Mutation of a gene encoding a putative chaperonin causes McKusick-Kaufman syndrome. Nat Genet. 2000;25(1):79-82.

41. Chang B, et al. In-frame deletion in a novel centrosomal/ciliary protein CEP290/NPHP6 perturbs its interaction with RPGR and results in early-onset retinal degeneration in the rd16 mouse. Hum Mol Genet. 2006;15(11):1847-1857.

42. McEwen DP, et al. Hypomorphic CEP290/NPHP6 mutations result in anosmia caused by the selective loss of $\mathrm{G}$ proteins in cilia of olfactory sensory neurons. Proc Natl Acad Sci U S A. 2007; 104(40):15917-15922.

43. Yen HJ, Tayeh MK, Mullins RF, Stone EM, Sheffield VC, Slusarski DC. Bardet-Biedl syndrome genes are important in retrograde intracellular trafficking and Kupffer's vesicle cilia function. Hum Mol Genet. 2006;15(5):667-677.

44. Badano JL, et al. Dissection of epistasis in oligogenic Bardet-Biedl syndrome. Nature. 2006; 439(7074):326-330.

45. Baye LM, et al. The N-terminal region of centrosomal protein 290 (CEP290) restores vision in a zebrafish model of human blindness. Hum Mol Genet. 2011;20(8):1467-1477.

46. Essner JJ, Amack JD, Nyholm MK, Harris EB, Yost HJ. Kupffer's vesicle is a ciliated organ of asymmetry in the zebrafish embryo that initiates left-right development of the brain, heart and gut. Development. 2005;132(6):1247-1260.

47. Colantonio JR, et al. The dynein regulatory complex is required for ciliary motility and otolith biogenesis in the inner ear. Nature. 2009; 457(7226):205-209.

48. Ross AJ, et al. Disruption of Bardet-Biedl syndrome ciliary proteins perturbs planar cell polarity in vertebrates. Nat Genet. 2005;37(10):1135-1140.

49. Fath MA, et al. Mkks-null mice have a phenotype resembling Bardet-Biedl syndrome. Hum Mol Genet. 2005;14(9):1109-1118.

50. Frolenkov GI, Belyantseva IA, Friedman TB, Griffith AJ. Genetic insights into the morphogenesis of inner ear hair cells. Nat Rev Genet. 2004; 5(7):489-498.

51. May-Simera HL, Ross A, Rix S, Forge A, Beales PL, Jagger DJ. Patterns of expression of Bardet-Biedl syndrome proteins in the mammalian cochlea suggest noncentrosomal functions. J Comp Neurol. 2009;514(2):174-188.

52. Besharse JC, Baker SA, Luby-Phelps K, Pazour GJ. Photoreceptor intersegmental transport and retinal degeneration: a conserved pathway common to motile and sensory cilia. Adv Exp Med Biol. 2003;533:157-164.

53. Kim J, Krishnaswami SR, Gleeson JG. CEP290 interacts with the centriolar satellite component PCM-1 and is required for Rab8 localization to the primary cilium. Hum Mol Genet. 2008;17(23):3796-3805.

54. Coppieters F, Lefever S, Leroy BP, De Baere E. CEP290, a gene with many faces: mutation overview and presentation of CEP290base. Hum Mutat. 2010;31(10):1097-1108

55. Schafer T, et al. Genetic and physical interaction between the NPHP5 and NPHP6 gene products. Hum Mol Genet. 2008;17(23):3655-3662.

56. Gorden NT, et al. CC2D2A is mutated in Joubert syndrome and interacts with the ciliopathy-associated basal body protein CEP290. Am J Hum Genet. 2008;83(5):559-571.

57. Kim JC, et al. MKKS/BBS6, a divergent chaperonin-like protein linked to the obesity disorder Bardet-Biedl syndrome, is a novel centrosomal component required for cytokinesis. J Cell Sci. 2005; 118(pt 5):1007-1020.

58. den Hollander AI, et al. Mutations in the CEP290 (NPHP6) gene are a frequent cause of Leber congenital amaurosis. Am J Hum Genet. 2006; $79(3): 556-561$

59. Ou G, Blacque OE, Snow JJ, Leroux MR, Scholey JM. Functional coordination of intraflagellar transport motors. Nature. 2005;436(7050):583-587.

60. Hudak LM, et al. The intraflagellar transport protein ift 80 is essential for photoreceptor survival in a zebrafish model of jeune asphyxiating thoracic dystrophy. Invest Ophthalmol Vis Sci. 2010;51(7):3792-3799.

61. Boldt K, et al. Disruption of intraflagellar protein transport in photoreceptor cilia causes Leber congenital amaurosis in humans and mice. J Clin Invest. 2011;121(6):2169-2180.

62. Krock BL, Perkins BD. The intraflagellar transport protein IFT57 is required for cilia maintenance and regulates IFT-particle-kinesin-II dissociation in vertebrate photoreceptors. J Cell Sci. 2008; 121(pt 11):1907-1915.

63. Sukumaran S, Perkins BD. Early defects in photoreceptor outer segment morphogenesis in zebrafish ift57, ift88 and ift172 Intraflagellar Transport mutants. Vision Res. 2009;49(4):479-489.

64. Lechtreck KF, Luro S, Awata J, Witman GB. HAtagging of putative flagellar proteins in Chlam$y$ domonas reinhardtii identifies a novel protein of intraflagellar transport complex B. Cell Motil Cytoskeleton. 2009;66(8):469-482.

65. Cuschieri A, Bannister LH. The development of the olfactory mucosa in the mouse: electron microscopy. J Anat. 1975;119(pt 3):471-498.

66. Hilborn RC. Chaos And Nonlinear Dynamics: An Introduction For Scientists And Engineers. Oxford, United Kingdom: Oxford University Press; 2004.

67. Yang J, Liu X, Yue G, Adamian M, Bulgakov O, Li T. Rootletin, a novel coiled-coil protein, is a structural component of the ciliary rootlet. J Cell Biol. 2002; 159(3):431-440.

68. Khanna H, et al. RPGR-ORF15, which is mutated in retinitis pigmentosa, associates with SMC1, SMC3, and microtubule transport proteins. J Biol Chem. 2005;280(39):33580-33587. 